\title{
Sequential infection with influenza $A$ virus followed by severe acute respiratory syndrome coronavirus 2 (SARS-CoV-2) leads to more severe disease and encephalitis in a mouse model of COVID-19.
}

Jordan J. Clark ${ }^{1 \dagger}$, Rebekah Penrice-Randal ${ }^{1 \dagger}$, Parul Sharma ${ }^{1}$, Anja Kipar ${ }^{1,2}$, Xiaofeng Dong ${ }^{1}$, Shaun H. Pennington ${ }^{3}$, Amy E. Marriott ${ }^{3}$, Stefano Colombo ${ }^{3}$, Andrew Davidson ${ }^{4}$, Maia Kavanagh Williamson 4 , David A. Matthews ${ }^{4}$, Lance Turtle ${ }^{5,6}$, Tessa Prince ${ }^{1}$, Grant L. Hughes ${ }^{7}$, Edward I. Patterson ${ }^{7}$, Ghada Shawli ${ }^{1}$, Krishanthi Subramaniam ${ }^{1}$, Jo Sharp ${ }^{8}$, Lynn McLaughlin ${ }^{9}$, En-Min Zhou'1,10, Joseph D. Turner ${ }^{3}$, Giancarlo Biagini ${ }^{3}$, Andrew Owen ${ }^{8}$, Julian A. Hiscox ${ }^{1,10,11}$, James P. Stewart ${ }^{1,10,12}$

${ }^{1}$ Department of Infection Biology \& Microbiomes, Institute of Infection, Veterinary and Ecological Sciences, University of Liverpool, UK.

2Laboratory for Animal Model Pathology, Institute of Veterinary Pathology, University of Zurich, Switzerland.

${ }^{3}$ Department of Tropical Disease Biology, Centre for Drugs and Diagnostics, Liverpool School of Tropical Medicine, Liverpool, UK.

${ }^{4}$ School of Cellular and Molecular Medicine, Faculty of Life Sciences, University of Bristol, Bristol, UK.

${ }^{5}$ Department of Clinical Infection Microbiology and Immunology and NIHR Health Protection Research Unit for Emerging and Zoonotic Infections, Institute of Infection, Veterinary and Ecological Sciences, University of Liverpool, UK.

${ }^{6}$ Tropical \& Infectious Disease Unit, Royal Liverpool University Hospital

${ }^{7}$ Departments of Vector Biology and Tropical Disease Biology, Centre for Neglected Tropical Disease, Liverpool School of Tropical Medicine, Liverpool, UK.

${ }^{8}$ Department of Pharmacology and Therapeutics, Centre of Excellence in Long-acting Therapeutics (CELT), University of Liverpool, UK.

${ }^{9}$ Biomedical Services Unit, University of Liverpool, UK.

${ }^{10}$ Department of Preventive Veterinary Medicine, Northwest A\&F University, Yangling, Shaanxi, China.

${ }^{11}$ Infectious Diseases Horizontal Technology Centre (ID HTC), A*STAR, Singapore.

${ }^{12}$ Department of Infectious Disease, University of Georgia, Georgia, USA.

${ }^{*}$ Corresponding authors: E. mail: j.p.stewart@liv.ac.uk; julian.hiscox@liverpool.ac.uk tThese authors contributed equally to this work 


\section{Abstract}

COVID-19 is a spectrum of clinical symptoms in humans caused by infection with SARS-CoV-2, a recently emerged coronavirus that has rapidly caused a pandemic. Coalescence of a second wave of this virus with seasonal respiratory viruses, particularly influenza virus is a possible global health concern. To investigate this, transgenic mice expressing the human ACE2 receptor driven by the epithelial cell cytokeratin-18 gene promoter (K18-hACE2) were first infected with IAV followed by SARS-CoV-2. The host response and effect on virus biology was compared to K18hACE2 mice infected with IAV or SARS-CoV-2 only. Infection of mice with each individual virus resulted in a disease phenotype compared to control mice. Although SARS-CoV-2 RNA synthesis appeared significantly reduced in the sequentially infected mice, these mice had a more rapid weight loss, more severe lung damage and a prolongation of the innate response compared to singly infected or control mice. The sequential infection also exacerbated the extrapulmonary manifestations associated with SARS-CoV-2. This included a more severe encephalitis. Taken together, the data suggest that the concept of 'twinfection' is deleterious and mitigation steps should be instituted as part of a comprehensive public health response to the COVID-19 pandemic. 


\section{Introduction}

Coronaviruses were once described as the backwater of virology but the last two decades have seen the emergence of three major coronavirus threats ${ }^{1}$. First, the emergence of severe acute respiratory syndrome coronavirus (SARS-CoV) in China in 2003. Second, Middle East respiratory syndrome coronavirus (MERS-CoV) in Saudi Arabia in 2012 and now SARS-CoV-2 originating in China in 2019. Whilst SARS-CoV was eradicated both MERS-CoV and SARS-CoV-2 represent current ongoing health threats, and a greater understanding is required to develop robust interventions for future emergent coronaviruses. Coronaviruses share similar genome architectures and disease profiles and generally cause respiratory and gastrointestinal illnesses ${ }^{1}$. However, some animal/avian coronaviruses can also affect other organ systems, causing, for example, demyelination and nephritis. The sheer scale of the COVID-19 outbreak has highlighted hitherto unexpected aspects of coronavirus infection in humans, including long term disease complications once the virus has been cleared.

Infection of humans with SARS-CoV-2 results in a range of clinical courses, from asymptomatic to severe infection and subsequent death in both at risk individuals but also a small proportion of otherwise healthy individuals across all age groups. Severe infection in humans is typified by cytokine storms ${ }^{2,3}$, pneumonia and kidney failure. Examination of post-mortem tissue reveals a disconnect between viral replication and immune pathology ${ }^{4}$. A range of other clinical signs also occur, including gastrointestinal symptoms such as vomiting, diarrhoea, abdominal pain and loss of appetite and loss of taste and smell (anosmia). A small number of patients have no overt respiratory symptoms at all. Typically, patients with severe COVID-19 present to hospital in the second week of illness. There is often a precipitous decline in respiratory function, without necessarily much in the way of "air hunger." Once intubated, these patients have unique ventilatory characteristics, where they can be ventilated with relatively low inspired oxygen concentrations but need high positive end expiratory pressures.

Respiratory infections in humans and animals can also be synergistic in which an initial infection can exacerbate a secondary infection or vice versa. When multiple pathogens are in circulation at the same time this can lead to cooperative or competitive forms of pathogen-pathogen interactions ${ }^{5}$. This was evident during the 
1918 Spanish influenza A virus outbreak (IAV) where secondary bacterial pneumonia was thought to be a leading cause of death ${ }^{6}$. Co-infections in other viral diseases, such as in patients with Ebola virus disease, have also been shown to contribute to the host response and outcome ${ }^{7}$. The onset of winter in the Northern Hemisphere has coincided with a second and possible seasonal wave of SARS-CoV-2 that is likely to be co-incident with other respiratory pathogens. In most temperate sites the usual seasonal respiratory coronavirus peak occurs either slightly before or simultaneously with the IAV and influenza B virus peaks, and this may occur with SARS-CoV-2. Generally, human coronaviruses display winter seasonality between the months of December and April and are not detected in summer months ${ }^{8}$. This is a similar pattern seen with influenza viruses. Between 11 to $41 \%$ of patients with normal human coronavirus infection test positive for other respiratory viruses ${ }^{8}$. Our hypothesis was that co-circulation of SARS-CoV-2 and IAV could lead to co-infection, and if so, this may exacerbate clinical disease and potentially outcome.

Previous work has shown co-infections are present in patients with severe coronavirus infection. For SARS-CoV co-circulation of human metapneumovirus was reported in an outbreak in Hong Kong. However, data suggested that outcomes were not different between patients with identified co-infections and those with SARS-CoV alone ${ }^{9}$. For MERS-CoV, four cases of co-infection with IAV were described, and although no data was presented on the severity of symptoms this sample size would be too small to allow any meaningful conclusions ${ }^{10}$. Post-mortem studies from patients with COVID19 in Beijing ( $n=85)$ identified IAV in $10 \%$ of patients, influenza B virus in $5 \%$ and RSV in $3 \%$ of patients, but the absence of a carefully selected control arm prohibits conclusions to be drawn ${ }^{11}$. Recently there have been several case reports of coinfections with IAV and SARS-CoV-2 in humans with severe outcomes ${ }^{12-17}$ with one study from the UK reporting that patients with a coinfection exhibited a $\sim 6$ times higher risk of death ${ }^{18}$. Whilst this suggests that co-infection is synergistic, this study also found that the risk of testing positive for SARS-CoV-2 was $68 \%$ lower among individuals who were positive for IAV infection, implying that the two viruses may competitively exclude each other ${ }^{18}$.

Whilst the analysis of post-mortem tissue is extremely informative in what may have led to severe coronavirus infection and death, the analysis of the disease in severe 
(but living cases) is naturally restricted by what tissues can be sampled (e.g. blood, nasopharyngeal swabs and bronchial alveolar lavages). Therefore, animal models of COVID-19 present critical tools to fill knowledge gaps for the disease in humans and for screening therapeutic or prophylactic interventions. Compatibility with a more extensive longitudinal tissue sampling strategy and a controlled nature of infection are key advantages of animal models ${ }^{19}$. Studies in an experimental mouse model of SARS-CoV showed that co-infection of a respiratory bacterium exacerbated pneumonia ${ }^{20}$. Different animal species can be infected with wild-type SARS-CoV-2 to serve as models of COVID-19 and these include mice, hamsters, ferrets, rhesus macaques and cynomolgus macaques. The K18-hACE2 transgenic (K18-hACE2) mouse, where hACE2 expression is driven by the epithelial cell cytokeratin-18 (K18) promoter, was developed to study SARS-CoV pathogenesis ${ }^{21}$. This mouse is now being used as a model that mirrors many features of severe COVID-19 infection in humans to develop understanding of the mechanistic basis of lung disease and to test pharmacological interventions ${ }^{22,23}$.

With the possibility of flu seasons concomitant with waves of SARS-CoV-2 infections there is an obvious public health concern about the possibility of enhanced morbidity and mortality in co-infected individuals. The aim of this work was to use an established pre-clinical model of COVID-19 to study the consequences of co-infection with SARSCoV-2 and IAV, defining the associated clinical, pathological and transcriptomic signatures. 


\section{Results}

\section{Sequential infection with IAV and SARS-CoV-2 leads to enhanced disease.}

To assess how co-infection with influenza virus affected COVID-19, the established K18-hACE2 mouse model of SARS-CoV-2 was utilised ${ }^{21}$. We sued a clinical isolate of SARS-CoV-2 (strain hCoV-19/England/Liverpool_REMRQ0001/2020)24. Importantly, sequence of the virus stock demonstrated that this isolate did not contain the recently observed deletion or mutations of the furin cleavage site in the $S$ protein ${ }^{25}$. A schematic of the experimental design is shown in Fig. 1A. Four groups of mice ( $\mathrm{n}$ $=8$ per group) were used. At day 0 , two groups were inoculated intranasally with $10^{2}$ PFU IAV (strain A/X31) and two groups with PBS. After three days, two groups were inoculated intranasally with $10^{4}$ PFU of SARS-CoV-2. This generated four experimental groups: Control, IAV only, SARS-CoV-2 and IAV + SARS-CoV-2 only (Fig. 1B). Control mice maintained their body weight throughout. Mice infected with IAV displayed a typical pattern of weight loss, reaching a nadir (mean 17\% loss) at 7 dpi before starting recovery. SARS-CoV-2-infected animals started to lose weight at day 7 (4 dpi) and carried on losing weight up to day 10 (mean 15\% loss). Mice infected with IAV then SARS-CoV-2 had a significantly-accelerated weight loss as compared with IAV-infected mice from day 4 which was most severe at day 6 (mean 19\%), followed by a recovery to day 8 (mean 14\% loss) before losing weight again (mean $17 \%$ loss) (Fig. 2A). As well as accelerated weight loss, IAV + SARS-CoV-2-infected mice exhibited more severe respiratory symptoms and a significantly more rapid mortality, (assessed by a humane endpoint of $20 \%$ weight loss) as compared with mice infected with either virus alone (Fig. 2B). 


\section{A}

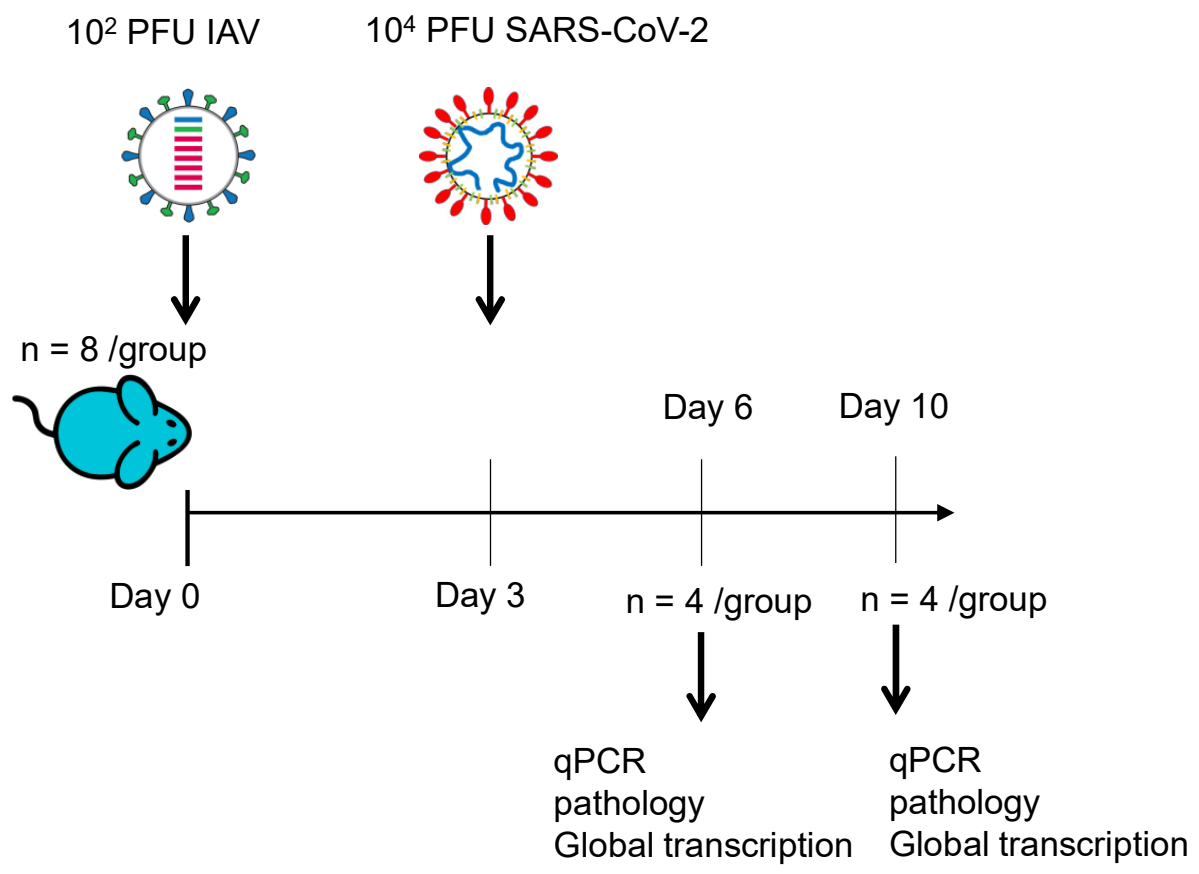

B

\begin{tabular}{|l|l|l|}
\hline Group & Day 0 & Day 3 \\
\hline Control & PBS & PBS \\
\hline IAV & IAV & PBS \\
\hline SARS-CoV-2 & PBS & SARS-CoV-2 \\
\hline IAV + SARS-CoV-2 & IAV & SARS-CoV-2 \\
\hline
\end{tabular}

Figure 1: (A) Schematic diagram of the experimental design for infection of K18-hACE2 mice sequentially with IAV strain A/X31 and SARS-CoV-2 (hCoV19/England/Liverpool_REMRQ0001/2020). B: Table showing exposures given to the four individual groups of mice 


\section{A}

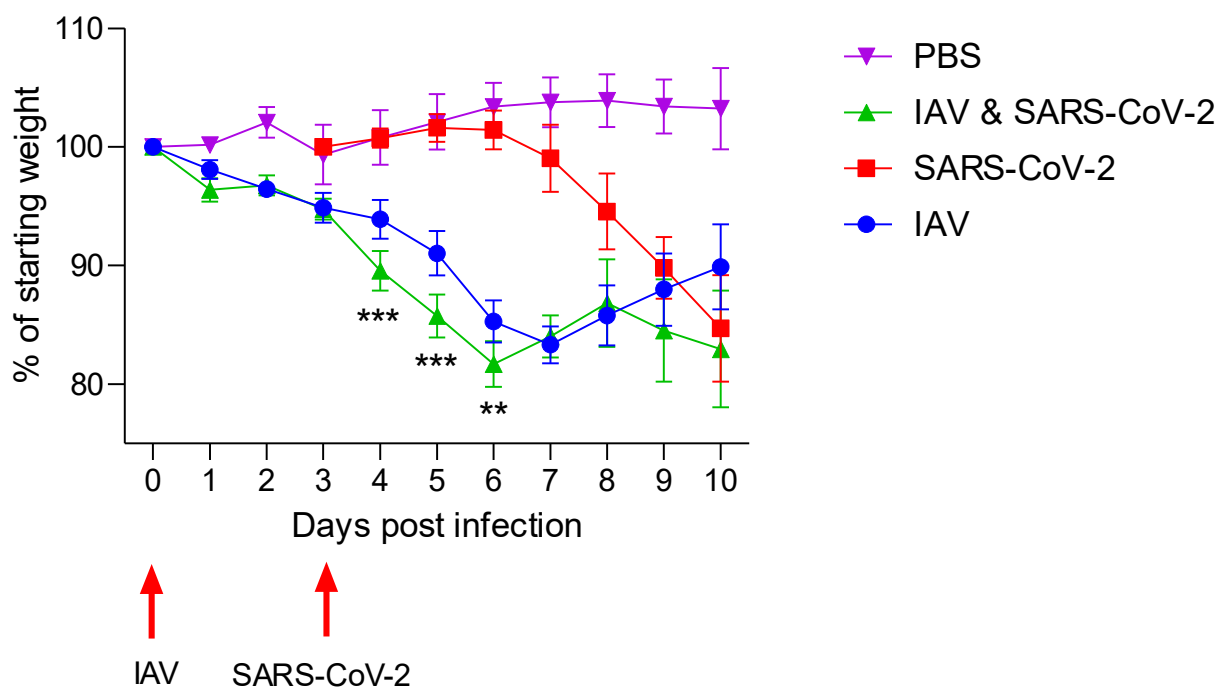

B

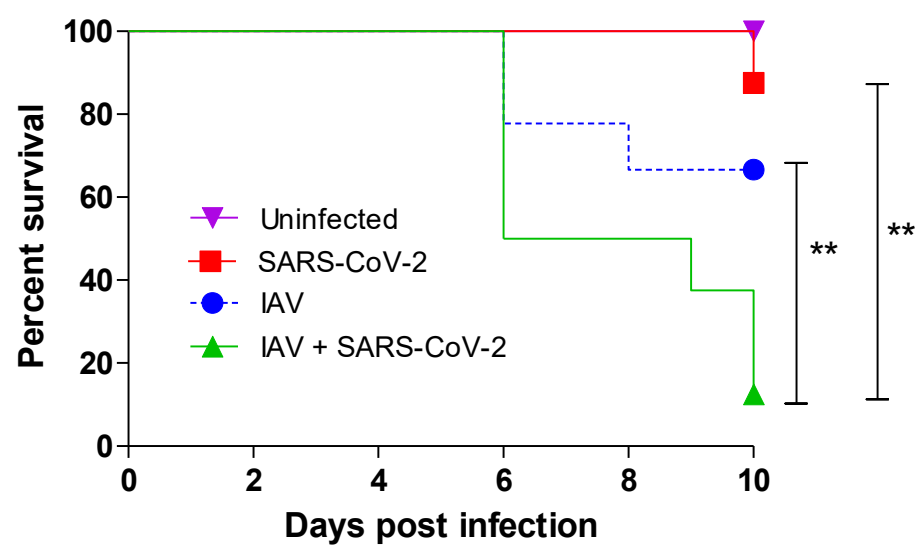

Figure 2: Co-infection with IAV and SARS-CoV-2 leads to enhanced weight loss and more rapid mortality. K18-hACE2 mice were challenged intranasally with IAV strain X31 (10 2 pfu) and 3 days later with $10^{4}$ PFU SARS-CoV-2 (A) Mice were monitored for weight loss at indicated time-points. $(n=8)$. Data represent the mean value \pm SEM. Comparisons were made using a repeated-measures two-way ANOVA (Bonferroni post-test). (B) Survival was assessed at indicated time points $(n=8)$. Comparisons were made using log-rank (MantelCox) test. ${ }^{* *}$ represents $\mathrm{P}<0.01$; ${ }^{* * *}$ represents $\mathrm{P}<0.001$ 


\section{Coinfection of SARS-COV-2 and IAV results in reduced SARS-COV-2 viral load at day 6 but not day 10 post IAV}

In order to determine whether the coinfection of SARS-CoV-2 and IAV was cooperative or competitive total RNA was extracted from the lungs of the K18-hACE2 mice and viral loads were quantified using qRT-PCR. At day 6 (3 dpi), the SARS-CoV2 infected mice exhibited 10,000-fold higher levels of viral load than at day 10 (7 dpi) (mean $6 \times 10^{12}$ vs $2.8 \times 10^{8}$ copies of $\mathrm{N} / \mu \mathrm{g}$ of RNA) indicating that peak viral replication takes place before the onset of symptoms at $4 \mathrm{dpi}$ (Fig, 3A). At this timepoint the mice infected with SARS-CoV-2 alone displayed significantly higher levels of viral RNA than the mice coinfected with IAV and SARS-COoV-2 (mean $6 \times 10^{12} \mathrm{vs} \sim 2 \times 10^{9}$ copies of $N / \mu g$ of RNA) (Fig. 3A). However, by day 10 the coinfected and singly infected mice exhibited nearly identical levels of SARS-CoV-2 RNA (mean $2 \times 10^{8}$ vs $8.1 \times 10^{8}$ copies of $N / \mu g$ of RNA) (Fig. 3A). The levels of infectious virus generally corresponded with the copies of N RNA, except at day 10, there was no infectious virus in mice infected with SARS-CoV-2 alone whereas the level of infectious virus in co-infected mice was similar at both day 6 and day $10\left(10^{2}\right.$ PFU/lung)(Fig. 3C) Conversely, at day 6, the mice infected with IAV alone showed similar levels of IAV RNA compared to the coinfected mice (mean $1.3 \times 10^{7}$ vs $1 \times 10^{7}$ copies of M/ $\mu$ g of RNA) and by day 10 both the singly infected mice and coinfected mice did not display any detectable IAV RNA, demonstrating similar levels of IAV clearance (Fig. 3D). In order to investigate viral replication qPCR was employed to quantify viral subgenomic mRNA (sgRNA) transcripts. Unlike viral genomes, sgRNAs are not incorporated into virions, and can therefore be utilised to measure active virus infection. The amount of sgRNA in the SARS-CoV-2 infected mice was concomitant with the viral load, appearing to be 100fold higher at day 6 (3dpi) than day 10 (7dpi) (mean $6.2 \times 10^{6}$ vs $5.4 \times 10^{4}$ copies of $E$ sgRNA $/ \mu$ g of RNA) (Fig. 3B). Similarly, the amount of sgRNA was significantly lower in the coinfected mice compared to the SARS-CoV-2 singly infected mice (mean 6.2 x $10^{6}$ vs $1.7 \times 10^{4}$ copies of E sgRNA/ $\mu$ g of RNA) however, by day 10 ( $7 \mathrm{dpi}$ ) both coinfected and singly infected mice displayed similar levels of sgRNA (mean $5.4 \times 10^{4}$

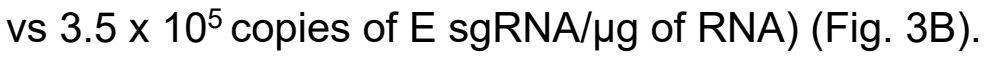


A

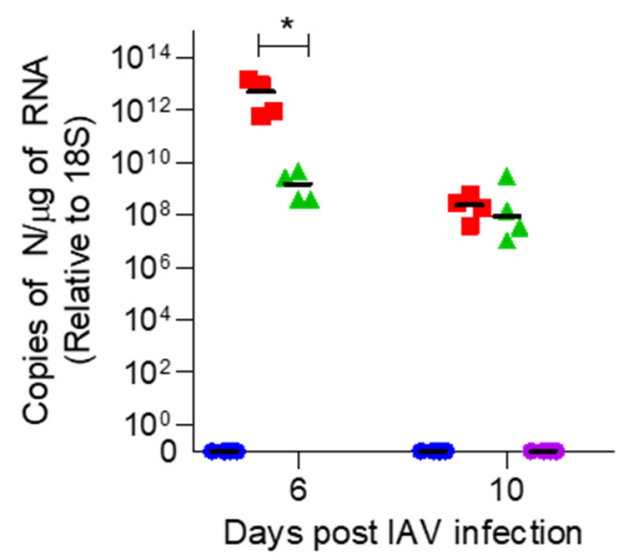

C

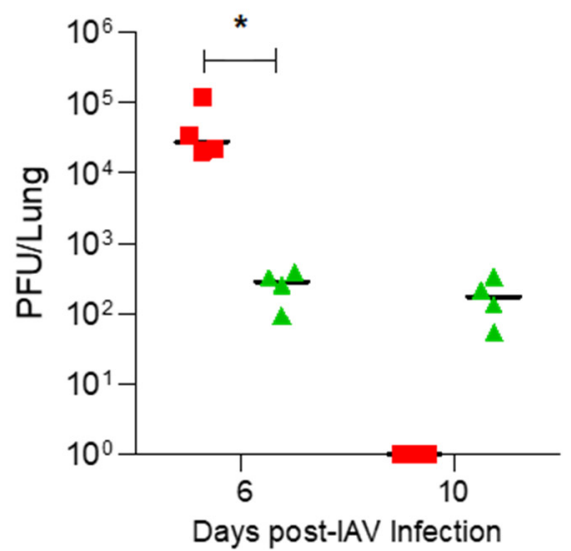

B

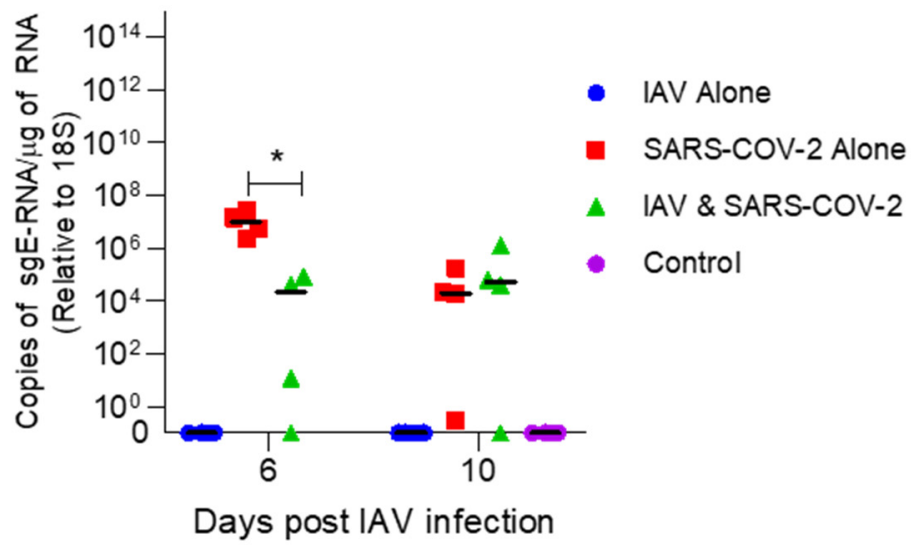

D

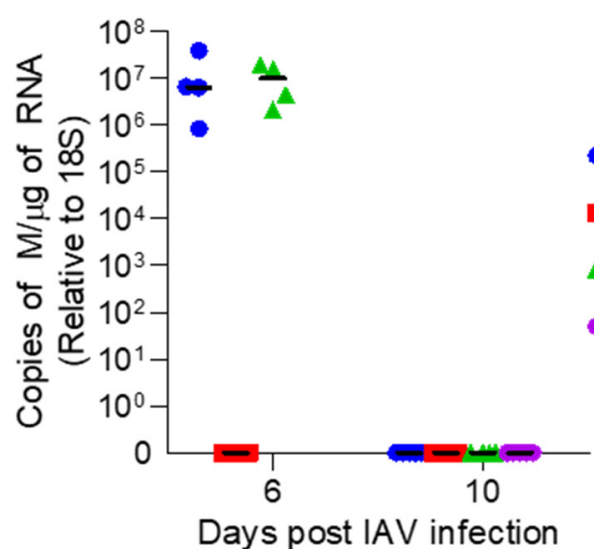

Figure 3: Viral loads and SARS-CoV-2 sgRNA levels in single and co-infected mice. K18-hACE2 mice were challenged intranasally with IAV strain X31 (102 pfu) and 3 days later with $10^{4}$ PFU SARS-CoV-2 $(n=4)$. RNA extracted from lungs was analysed for virus levels by qRT-PCR. Assays were normalised relative to levels of 18S RNA. Data for individual animals are shown with the median value represented by a black line (A) SARS-CoV-2 viral load was determined using qRT-PCR for the N gene. (B) Levels of SARS-CoV-2 sub-genomic RNA (sgRNA) for the E gene. (C) SARS-CoV-2 titre was determined by plaque assay on Vero E6 cells (D) IAV load was determined using RT-PCR for the M gene. Comparisons were made using two-way ANOVA (Bonferroni post-test). ${ }^{*}$ represents $p<0.05$

\section{Co-infection leads to complementary and enhanced pathological processes}

Transgenic mice carrying the human ACE2 receptor under the control of the keratin 18 promoter (K18-hACE2) have been reported as a suitable COVID-19 model ${ }^{22}$. As a basis for the assessment of the effect of IAV and SARS-CoV-2 in these mice, a histological examination of major organs/tissues was performed. This confirmed that the transgenic approach had not resulted in phenotypic changes. Comparative staining of wild type and K18-hACE2 mice for ACE2, using an antibody against human 
ACE2 that also cross-reacts with mouse ACE2, also confirmed that transgenesis had not altered the ACE2 expression pattern: in the lung, ACE2 was found to be expressed by respiratory epithelial cells and very rare type II pneumocytes (Supplementary Fig. S1 A, B). Expression was also seen in endothelial cells in brain capillaries (Supplementary Fig. S2 A2, A3) and liver sinusoids and in renal tubular epithelial cells). The expression was not substantially affected by viral infection (Supplemental Fig. S1C-F; Fig. S2 B, C).

At 6 days post IAV infection, the transgenic mice exhibited the pulmonary changes typically seen in mice after IAV X31 infection at this time point. We observed epithelial cell degeneration and necrosis in several bronchioles which also contained debris in the lumen (Fig. 4 B). There were occasional small focal peribronchial areas where alveoli also exhibited necrotic cells (Fig. 4 B). IAV antigen was found in epithelial cells in bronchi and bronchioles, in type I and II pneumocytes in affected alveoli, and in few randomly distributed individual type II pneumocytes (data not shown). Vessels showed evidence of lymphocyte recruitment, vasculitis and perivascular lymphocyte infiltration. Comparative assessment of the lungs in wild type mice at the same time point post infection confirmed that the genetic manipulation indeed had no effect on the response of mice to IAV infection (data not shown). At the comparative time point, SARS-CoV-2 single infection (day 6, 3 dpi) was associated with mild changes, represented by a mild increase in interstitial cellularity, evidence of type II pneumocyte activation (Fig. 4C, 5A), occasional desquamated alveolar macrophages/type II pneumocytes and single erythrocytes in alveolar lumina, and a multifocal, predominantly perivascular mononuclear infiltration with recruitment of leukocytes into vascular walls (vasculitis) (Fig. 4 D). Infiltrating cells were mainly macrophages, with T cells mainly around vessels and a low number of disseminated B cells (Fig. 5); macrophages and $T$ cells were also found to emigrate from veins (Fig. 5D, E). Viral antigen was found in multifocal patches of individual to large groups of alveoli, randomly distributed throughout the parenchyma (Fig. 5B). Viral antigen was seen in type I and type II pneumocytes and in endothelial cells in capillaries and small vessels in septa (Fig. 5C). However, viral antigen was not detected within bronchiolar epithelial cells (Fig. 5B). Double infection at this time point, i.e. 6 days after IAV infection and 3 days after SARS-CoV-2 infection was associated with histological changes almost identical to those induced by IAV, although they appeared to be slightly more extensive (Fig. 4 E, F). IAV antigen expression had a distribution and extent similar to that seen 
in single IAV infection at the same time point. It was seen in epithelial cells in bronchi and bronchioles, in type I and II pneumocytes in affected alveoli, and in few randomly distributed individual type II pneumocytes (Fig. 6B). SARS-CoV-2 expression was less intense than in SARS-CoV-2-only infected mice. Viral antigen was observed in random individual or small groups of alveoli (Fig. 6C), in type I and II pneumocytes and vascular endothelial cells (Fig. 6C inset).

Four days later, at the endpoint of the experiment, i.e. at 10 days after IAV infection and 7 days of SARS-CoV-2 infection, the histopathological features had changed. Single IAV infection had by then almost entirely resolved, however, the lungs exhibited changes consistent with a regenerative process, i.e. mild to moderate hyperplasia of the bronchiolar epithelium with adjacent multifocal respiratory epithelial metaplasia/type II pneumocyte hyperplasia, together with mild to moderate lymphocyte dominated perivascular infiltration (Fig. 6A). Interestingly, the hyperplastic epithelium was found to lack ACE2 expression (Supplemental Fig. S1 E). At this stage, the effect of SARS-CoV-2 infection was more evident. Single infection had resulted in multifocal areas with distinct type II pneumocyte activation and syncytial cell formation (Fig. 7B), mononuclear infiltration and mild to moderate lymphocyte-dominated vasculitis and perivascular infiltration. There were also a few focal areas of mild desquamative pneumonia with intra-alveolar macrophages/type II pneumocytes, oedema and fibrin deposition (Fig. 7C). Macrophages and T cells dominated in the infiltrates (Fig. 7D, E), whereas B cells were found disseminated in low numbers (Fig. 7F). The SARS-CoV-2 associated changes were also observed in the double infected mice (Fig. 8C-F), where they were generally more pronounced (Fig. 8B, C) and present alongside equally pronounced regenerative changes attributable to IAV infection (moderate hyperplasia of the bronchiolar epithelium with adjacent multifocal respiratory epithelial metaplasia/type II pneumocyte hyperplasia; Fig. 8A). Also in this group of animals, macrophages were the dominant infiltrating cells. However, the number of $\mathrm{T}$ cells was comparatively low (Fig. 8D, E). B cells were generally rare, but occasionally formed small aggregates in proximity to areas of type II pneumocytes hyperplasia (Fig. 8F). Interestingly, the pattern of viral antigen expression had not changed with time; it was detected in type I and II pneumocytes of unaltered appearing alveoli (data not shown). 
In two of the four single SARS-CoV-infected and three of the four double infected mice at the later time point (7 days post SARS-CoV-2 infection), we observed a mild or moderate non-suppurative meningoencephalitis mainly affecting the midbrain and brainstem (Fig. 9). This was more severe in the double infected mice, where the perivascular infiltrates contained degenerate leukocytes and appeared to be associated with focal loss of integrity of the endothelial cell layer (Fig. 9B).

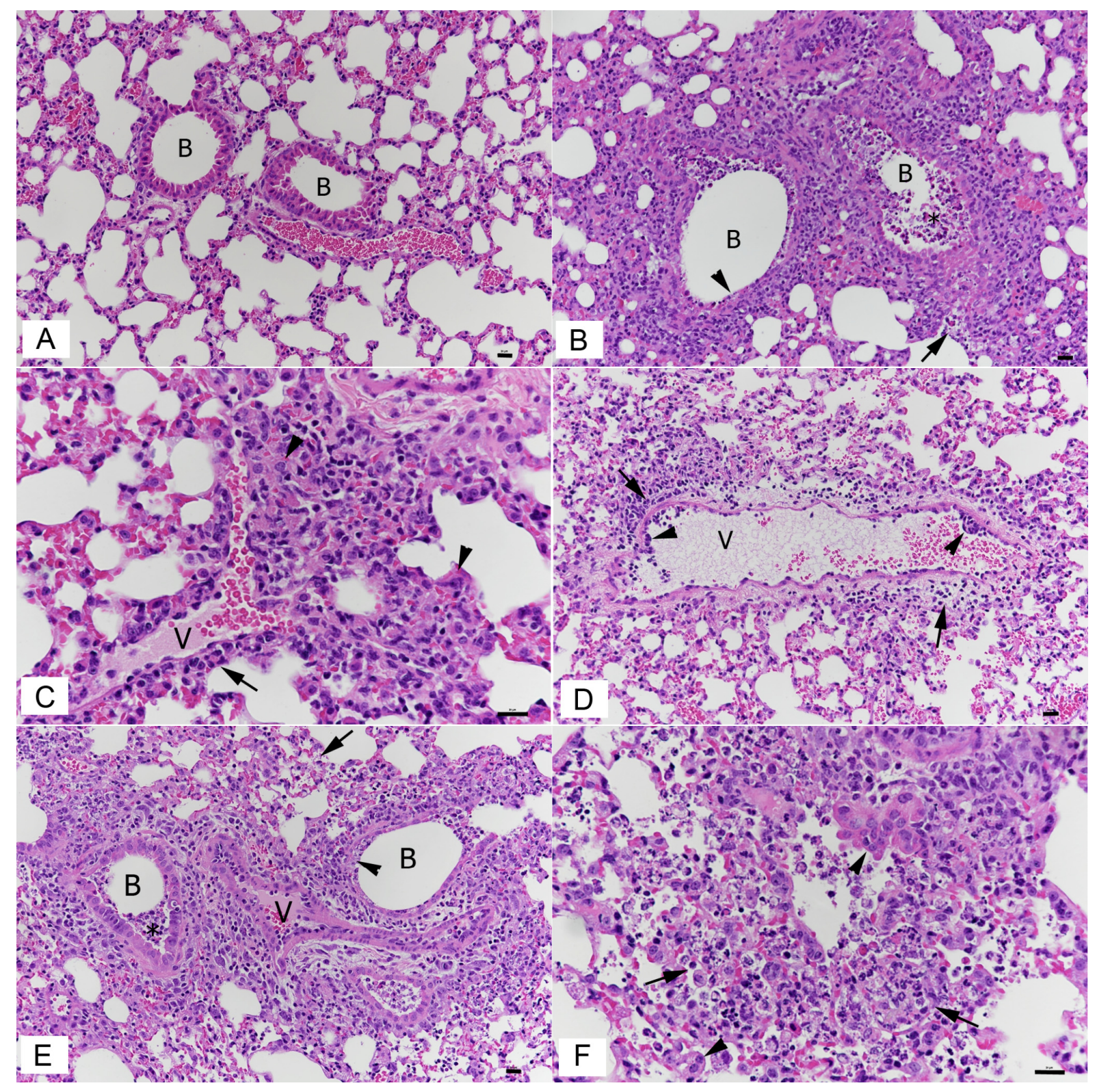

Figure 4: Lungs, K18-hACE2 transgenic mice, after mock infection or at day 6 post infection with IAV and day 3 post infection with SARS-CoV-2 in single and double infections. A. Mock infected control animal. Normal lung. B. IAV-infected animal; 6 dpi. Bronchioles (B) exhibit necrosis (arrowhead) of a variable amount of epithelial cells and contain degenerate cells in the lumen $\left(^{*}\right)$. The parenchyma adjacent to affected bronchioles often exhibits individual alveoli with necrotic epithelial cells (arrow). C, D. SARS-CoV-2-infected animal; 3 dpi. The parenchyma exhibits multifocal activation of type II pneumocytes (C: arrowheads), and there 
bioRxiv preprint doi: https://doi.org/10.1101/2020.10.13.334532; this version posted February 15, 2021. The copyright holder for this

preprint (which was not certified by peer review) is the author/funder, who has granted bioRxiv a license to display the preprint in perpetuity. It is made available under aCC-BY 4.0 International license.

is evidence of vasculitis, represented by leukocyte infiltration of vessel (V) walls (D: arrowheads) and perivascular infiltrates (arrows). E, F. IAV (6 dpi) and SARS-CoV-2 (3 dpi) double infection. The IAV-associated changes, with necrosis of bronchiolar epithelial cells (E: arrowhead), debris in bronchiolar lumina $\left({ }^{*}\right)$, focal necrosis of alveolar epithelial cells (arrows) as well some activation and hyperplasia of type II pneumocytes ( $F$ : arrowheads), dominate the histological picture. B - bronchiole; V - vessel. HE stain; Bars represent $20 \mu \mathrm{m}$.

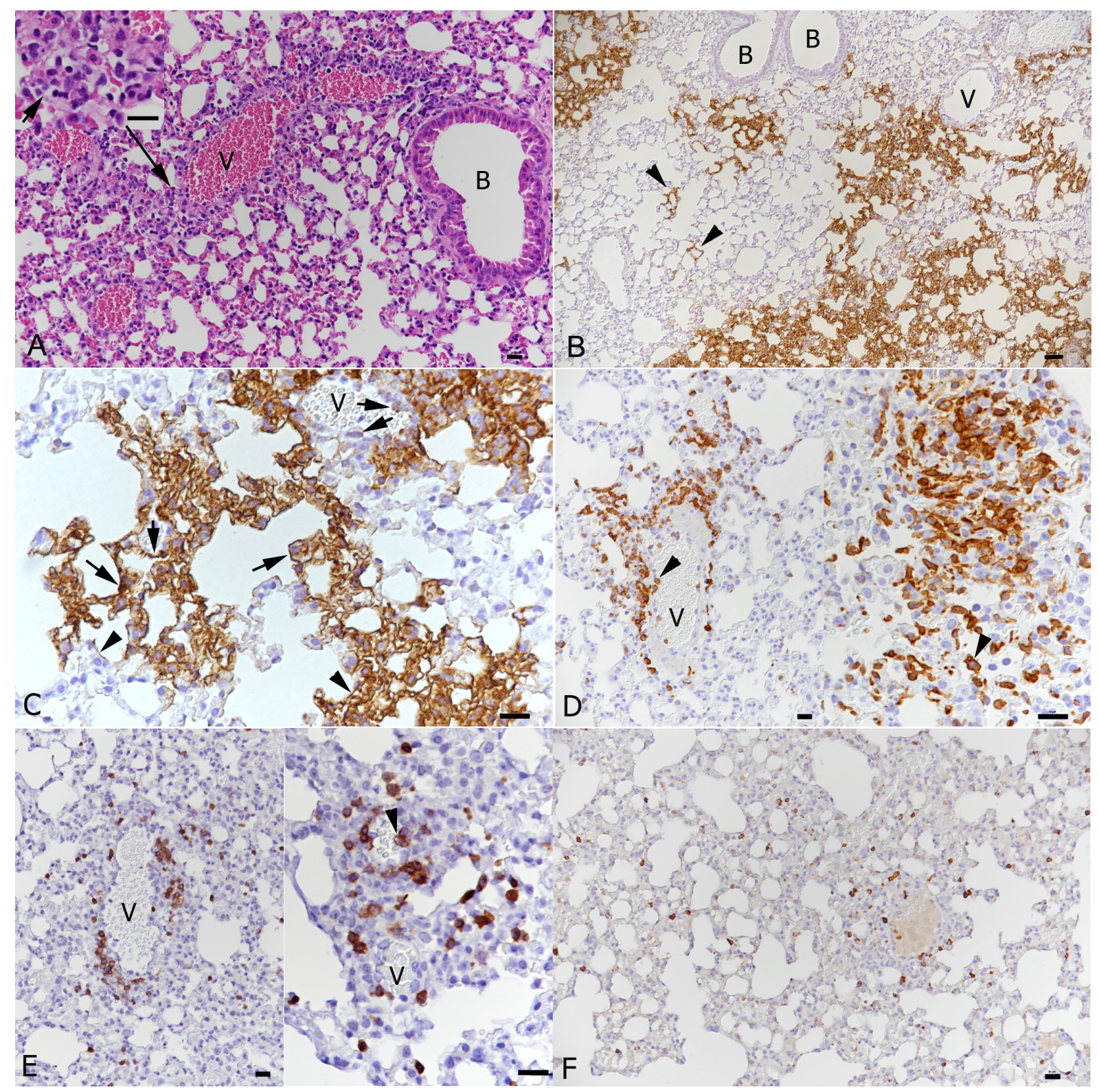

Figure 5: Lungs, K18-hACE2 transgenic mice, at day 3 post infection with SARS-CoV-2. A. There is mild perivascular mononuclear infiltration, and the parenchyma exhibits mild multifocal activation of type II pneumocytes (inset). B, C. Staining for SARS-CoV-2 antigen reveals random multifocal areas of SARS-CoV-2 infection, affecting both individual alveoli (B: arrowheads) and large parenchymal areas. Viral antigen expression is seen in type I pneumocytes (C: arrowheads), type II pneumocytes (C: arrows) and vascular endothelial cells (C: small, short arrows). D. Staining for macrophages (Iba-1+) shows recruitment from (left image, arrowhead: monocytes attached to the endothelium of a vein) and accumulation of monocytes around veins, macrophage accumulation in the parenchyma and desquamation of alveolar macrophages (right image, arrowhead). E. T cells (CD3+) are less numerous than macrophages and are mainly found in the perivascular infiltrates. They are also recruited from 
the blood (right image, arrowhead). F. B cells (CD45R/B220+) are seen in low numbers, and disseminated in the parenchyma. B - bronchiole; V - vessel. HE stain; immunohistology, hematoxylin counterstain. Bars represent $20 \mu \mathrm{m}$.

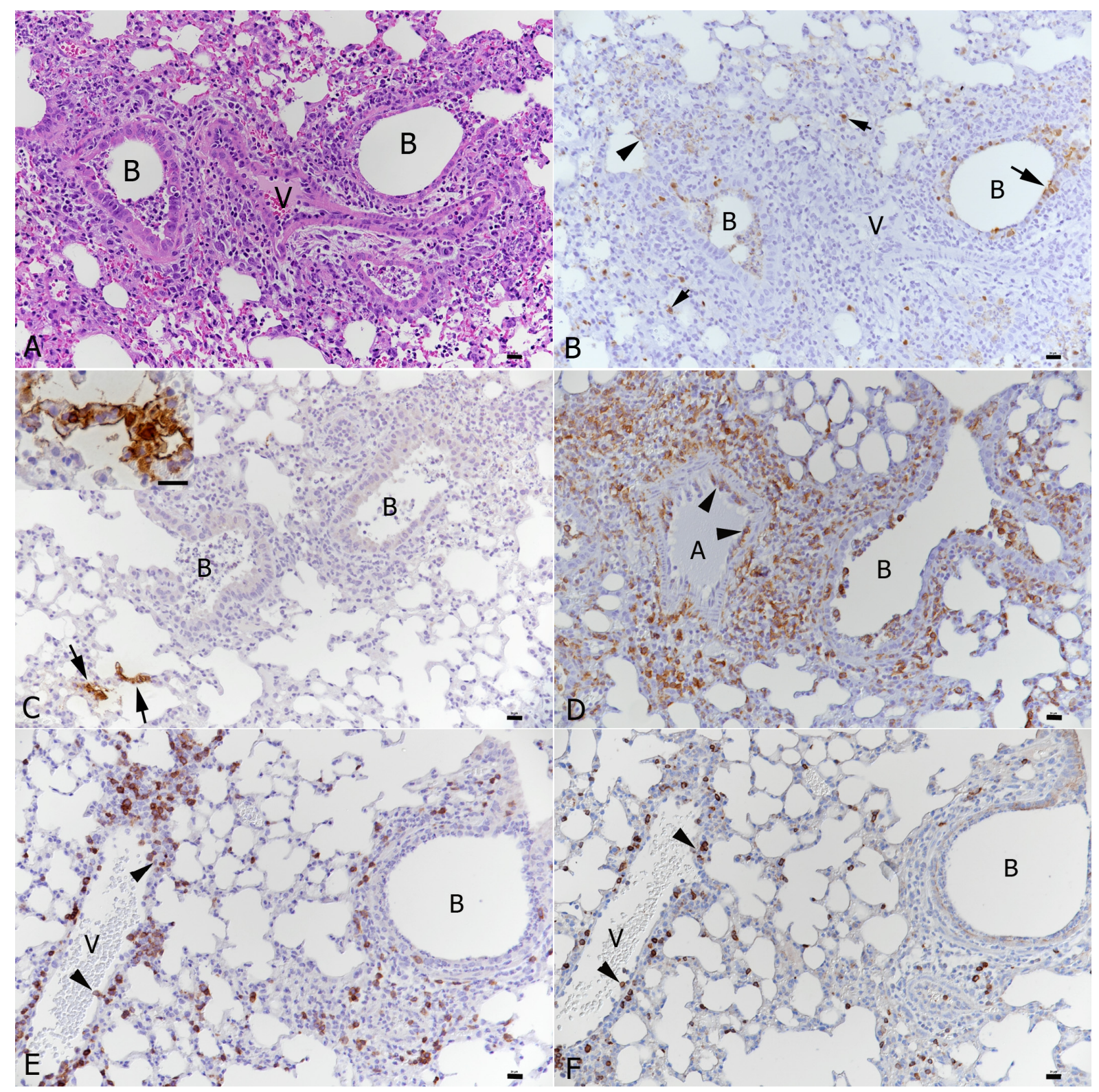

Figure 6: Lungs, K18-hACE2 transgenic mice, at day 6 post infection with IAV and day 3 post infection with SARS-CoV-2 in double infection. A. The IAV-associated changes dominate (see also Fig. 4E). B. This is confirmed by staining for IAV antigen which is detected in bronchiolar epithelial cells (arrow), occasional type I pneumocytes (arrowhead) and disseminated type II pneumocytes (short, small arrows). C. SARS-CoV-2 infection is seen in areas not affected by IAV-induced changes (B: bronchioles with IAV changes) and mainly in individual alveoli where both type I and type II pneumocytes are found to express viral antigen (inset). D. Macrophages (Iba-1+) are abundant around affected bronchioles and in the exudate in the bronchiolar lumen, and are recruited from the blood into the perivascular infiltrates (arrowheads: rolling and emigrating monocytes). E. T cells (CD3+) are recruited in moderate numbers from the blood (arrowheads) into the perivascular infiltrates. F. B cells (CD45R/B220+) are recruited in low numbers from the blood (arrowheads) into the perivascular infiltrates. B - bronchiole; $\mathrm{V}$ vessel. HE stain; immunohistology, hematoxylin counterstain. Bars represent $20 \mu \mathrm{m}$. 


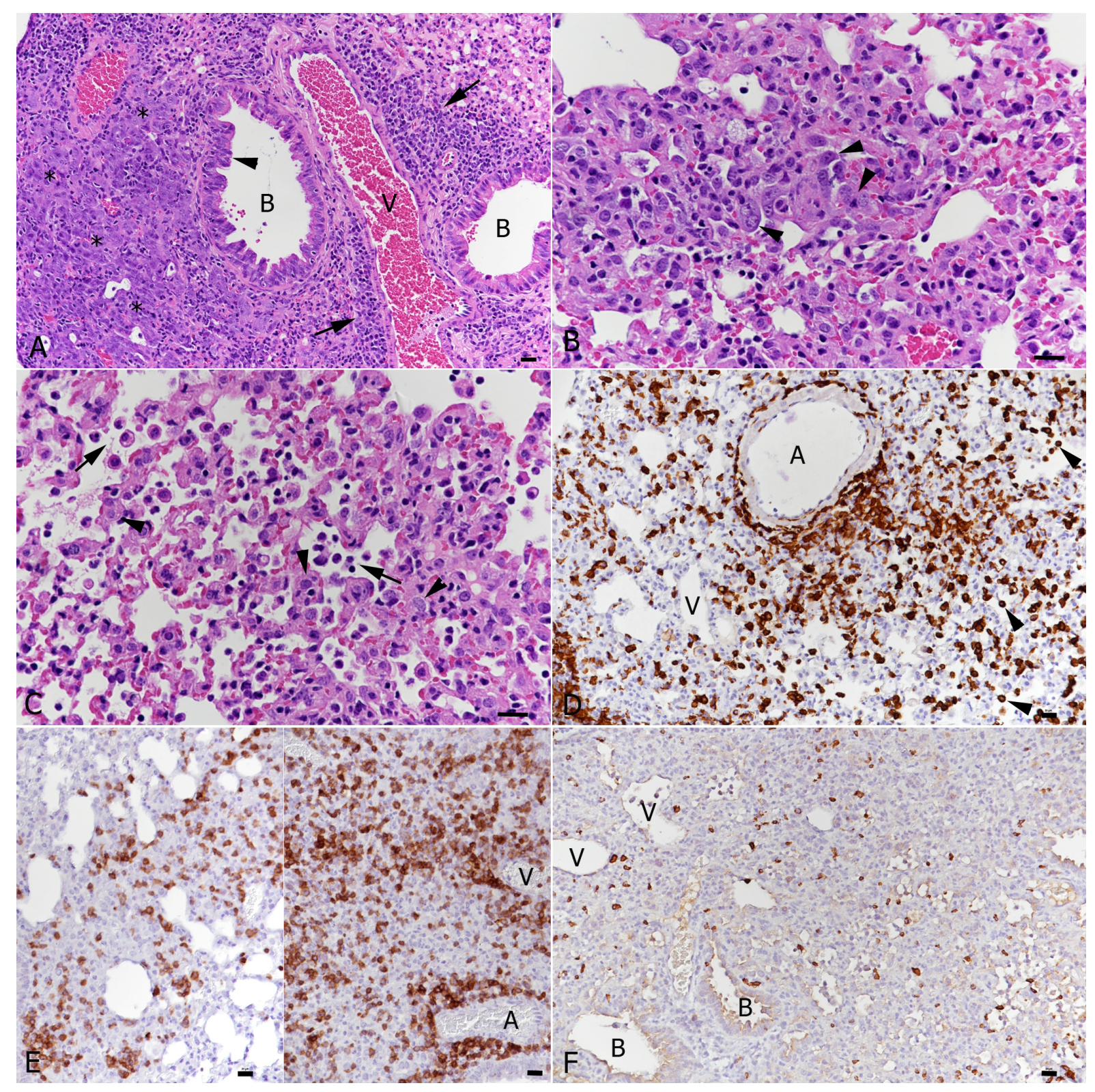

Figure 7: Lungs, K18-hACE2 transgenic mice, at day 10 post infection with IAV and day 7 post infection with SARS-CoV-2 in single infections. A. IAV-infected animal; 10 dpi. Bronchioles (B) exhibit epithelial cell hyperplasia (arrowhead) and there is type II pneumocyte hyperplasia $\left(^{*}\right)$ in the adjacent parenchyma. Vessels (V) exhibit variably intense lymphocytedominated perivascular infiltrates (arrows). B-F. SARS-CoV-2-infected animal; $7 \mathrm{dpi}$. B. There are abundant activated type II pneumocytes which also show syncytia formation (arrowheads). C. There are also focal changes consistent with desquamative pneumonia, with desquamation of alveolar macrophages/type II pneumocytes (arrows) and type II pneumocyte activation (arrowheads). D. Macrophages (Iba-1+) are abundant in perivascular infiltrates and within the altered parenchyma. E. T cells (CD3+) are also abundant in the parenchymal infiltrates. F. B cells (CD45R/B220+) are seen in low numbers disseminated in the parenchymal infiltrates. A - artery; B - bronchiole; V - vessel. HE stain; immunohistology, hematoxylin counterstain. Bars represent $20 \mu \mathrm{m}$. 


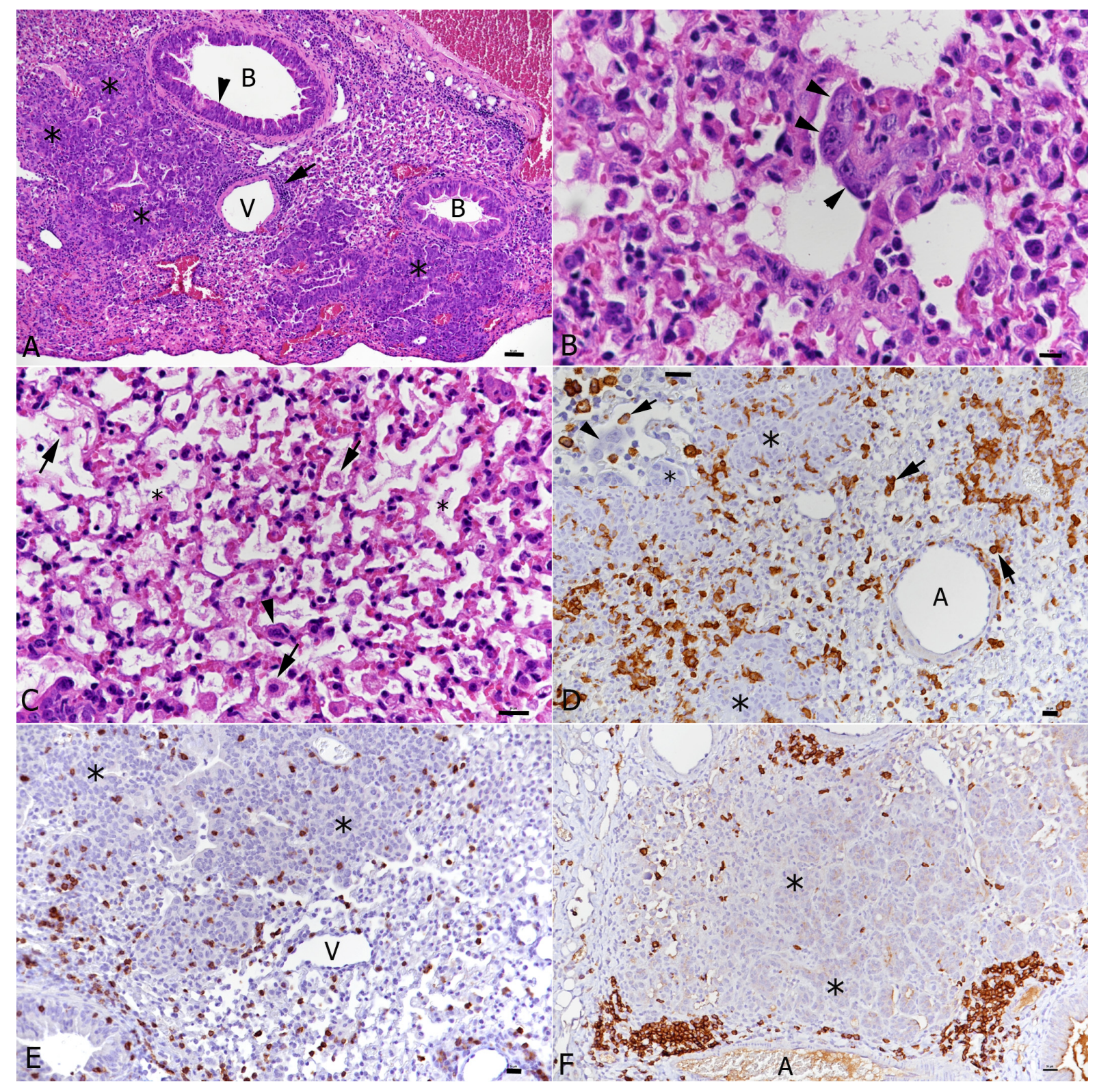

Figure 8: Lungs, K18-hACE2 transgenic mice, at day 10 post infection with IAV and day 7 post infection with SARS-CoV-2 in double infection. A. There are abundant changes consistent with those seen in single IAV-infected mice, i.e. epithelial cell hyperplasia in bronchioles (B), multifocal type II pneumocyte hyperplasia $\left({ }^{*}\right)$, and perivascular lymphocyte dominated infiltrates (arrow). B, C. Changes attributable to SARS-CoV-2 infection. These comprise type II pneumocyte activation and syncytia formation ( $\mathrm{B}$ : arrowheads) and desquamative pneumonia (C), with desquamation of alveolar macrophages/type II pneumocytes (arrows) and type II pneumocyte activation (arrowheads). In more severe cases, alveoli occasionally contain fibrin and hyaline membranes $\left(^{*}\right)$. D. Macrophages (lba-1+) form focal parenchymal infiltrates and are found around areas of type II pneumocyte hyperplasia $\left({ }^{*}\right)$. There are also desquamating alveolar macrophages (Iba-1+; arrows). The lack of Iba-1 expression in syncytial cells confirms that they are type II pneumocytes (inset: arrowhead). E. T cells (CD3+) are present in moderate numbers throughput the infiltrates and around areas of type II pneumocyte hyperplasia $\left({ }^{*}\right)$ F. B cells (CD45R/B220+) form occasional small aggregates in proximity to areas of type II pneumocyte hyperplasia $\left(^{*}\right)$. A - artery; B bronchiole; $V$ - vessel. HE stain; immunohistology, hematoxylin counterstain. Bars represent $20 \mu \mathrm{m}$. 


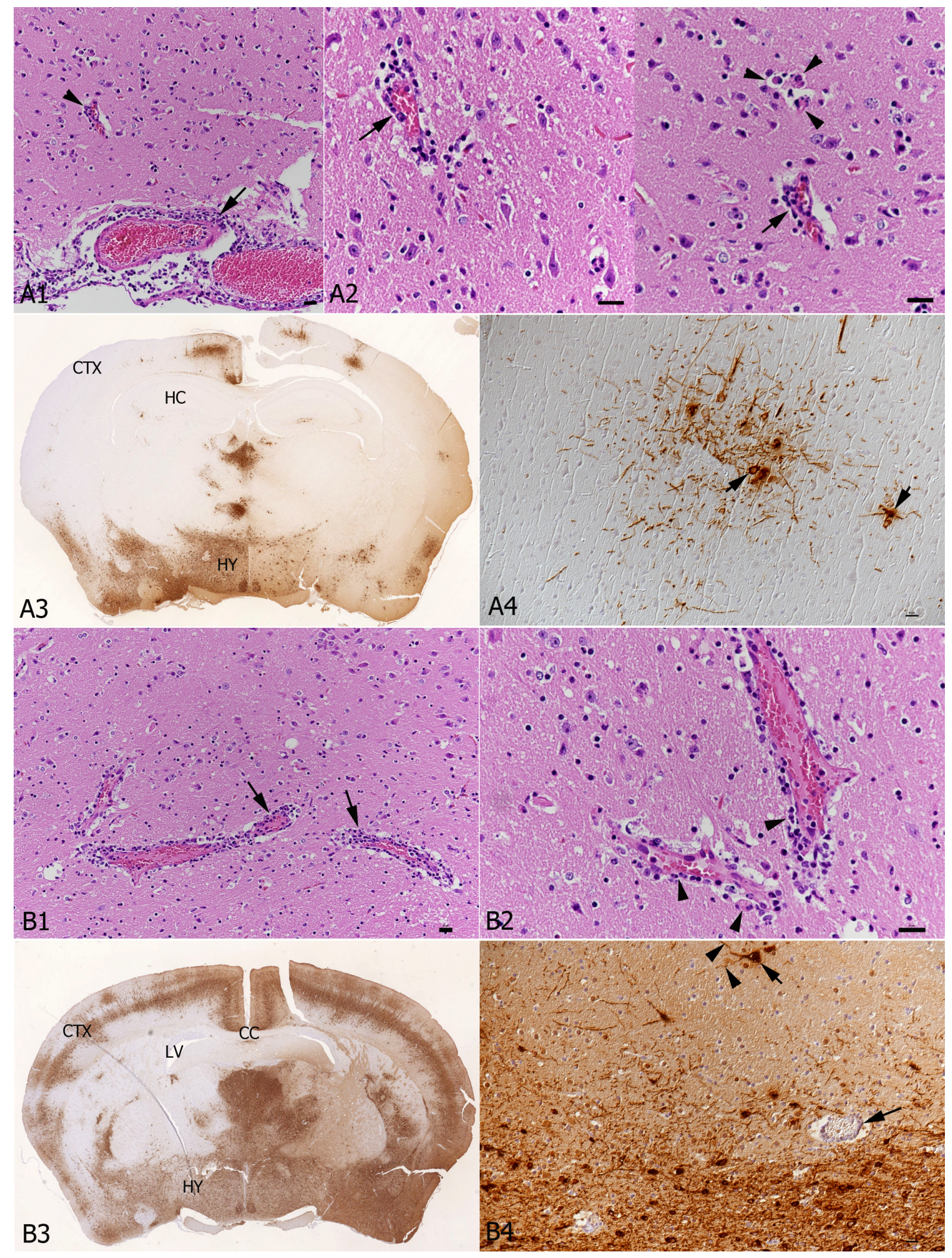

Figure 9: Brain, K18-hACE2 transgenic mice, after day 7 post single infection with SARSCoV-2 or at day 10 post infection with IAV and day 7 post infection with SARS-CoV-2 in double infections. A. SARS-CoV-2 single infection. A1, A2. Hypothalamus. A1. Vessels in the leptomeninx (arrow) and in the brain parenchyma (arrowhead) exhibit mild perivascular mononuclear infiltrations, consistent with mild non-suppurative meningoencephalitis. A2. Higher magnification highlighting the one-layered perivascular infiltrate (arrows). There is 
evidence of focal neuronal degeneration (right image; arrowheads). A3. Coronal section at the level of the hippocampus (HC), showing extensive SARS-CoV-2 antigen expression in the hypothalamus (HY) and bilateral patchy areas with positive neurons also in the cortex (CTX). A4. A higher magnification of a focal area with SARS-CoV-2 expression shows that infection is in the neurons (arrowheads). B. IAV and SARS-CoV-2 double infected K18-hACE2 transgenic mouse. B1, B2. The perivascular mononuclear infiltrate is slightly more intense than in the SARS-CoV-2 single infected mouse (B1: arrows), consistent with a moderate nonsuppurative encephalitis. Among the perivascular infiltrate are several degenerate cells (B2: arrowheads). B3. Coronal section at the level of the corpus callosum (CC), showing extensive widespread bilateral SARS-CoV-2 antigen expression (HY: hypothalamus; CTX: cortex; LV: left ventricle). HE stain and immunohistology, hematoxylin counterstain. Bars represent 20 $\mu \mathrm{m}$.

\section{Distinct transcriptional signatures are associated with infection}

The transcriptional profile of lung samples can provide a window on the host response to infection for a respiratory pathogen. Therefore, lung samples were taken at Day 6 and Day 10 post IAV infection from all four groups of mice (Fig. 1B). Total RNA was purified from cells and both host and viral mRNA (and genomic RNA in the case of SARS-CoV-2) were sequenced using the Oxford Nanopore oligo-dT cDNA synthesis approach to identify and quantify mRNA. A multiplex of 5-10 sequencing libraries were loaded onto a flow cell and sequenced on an Oxford Nanopore GridION for up to 72 hours.

Genes were counted against the Mus musculus annotated genome using Salmon ${ }^{26}$. Gene counts were normalised using the edgeR package before identifying differentially expressed genes using the transcription profile from mock infected mice as the control profile. A total of 970 differentially expressed gene transcripts were observed in comparison to mock infected animals out of a total of 3495 gene transcripts identified. Principle component analysis (PCA) revealed overlapping transcriptional profiles between infection groups (Fig. 10A). Overlapping signatures were likely to be indicative of the non-specific anti-viral response. Contrast matrices were made between mice that were coinfected versus mice that were mock infected and mice that were singly infected (Table 1). The transcriptomic profile in mice 10 days post infection with IAV showed overlap with the healthy controls, consistent with resolution of infection and regeneration seen in the pathology (Supplementary Fig S3). The data indicated that coinfection at day 10 versus IAV day 10 had more differences 
bioRxiv preprint doi: https://doi.org/10.1101/2020.10.13.334532; this version posted February 15,2021 . The copyright holder for this

preprint (which was not certified by peer review) is the author/funder, who has granted bioRxiv a license to display the preprint in perpetuity. It is made available under aCC-BY 4.0 International license.

with 36 gene transcripts at higher abundance, highlighted in the top 75 differentially expressed genes (Fig. 10B,C and Supplementary Figure S4). 
Table 1: Number off differentially expressed genes with an FDR value less than 0.05 and a log2 fold change more than 2 and less than -2 compared to mock infected mice. Coinfection day 6 and day 10 were compared to day 6 and 10 of individual IAV and SARS-CoV-2 infection.

$\begin{array}{cccclll}\text { IAVDay } 6 & \text { IAV } & \text { Day } & \text { SARS-Cov- } & \text { SARS- } & \text { Coinfection } & \text { Coinfection } \\ & 10 & 2 \text { Day } 6 & \text { CoV-2 Day } & \text { Day } 6 & \text { Day } 10\end{array}$

\begin{tabular}{l|llllll}
\hline Mock & 172 & 79 & 141 & 150 & 188 & 120 \\
& 38 & 24 & 5 & 37 & 52 & 24 \\
Coinfection & 2 & - & 7 & - & - & - \\
Day 6 & 4 & & 19 & & & - \\
Coinfection & - & 36 & - & 9 & - & - \\
Day 10 & & 6 & & 3 & &
\end{tabular}


bioRxiv preprint doi: https://doi.org/10.1101/2020.10.13.334532; this version posted February 15, 2021. The copyright holder for this

preprint (which was not certified by peer review) is the author/funder, who has granted bioRxiv a license to display the preprint in perpetuity. It is made available under aCC-BY 4.0 International license.

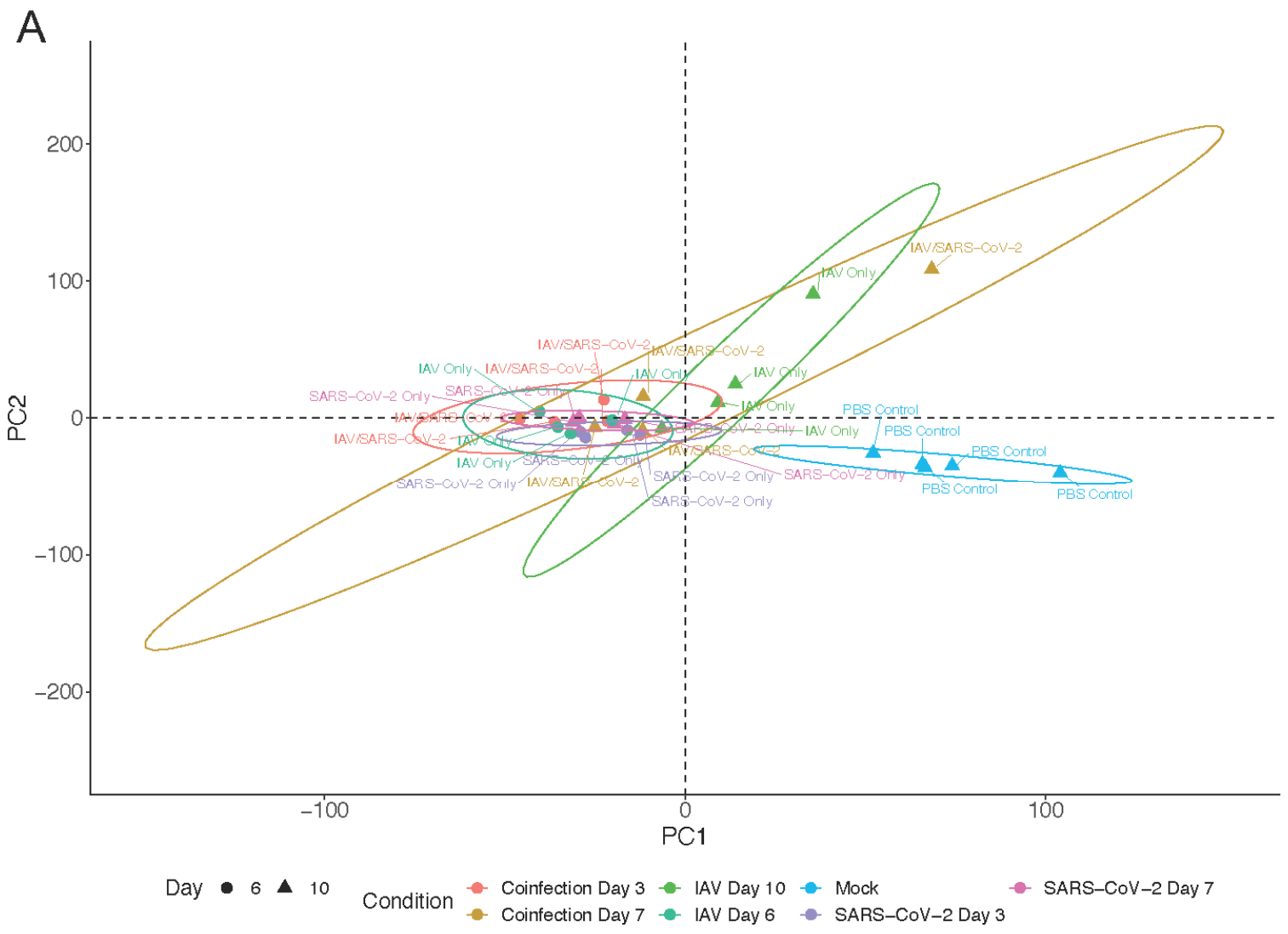

B

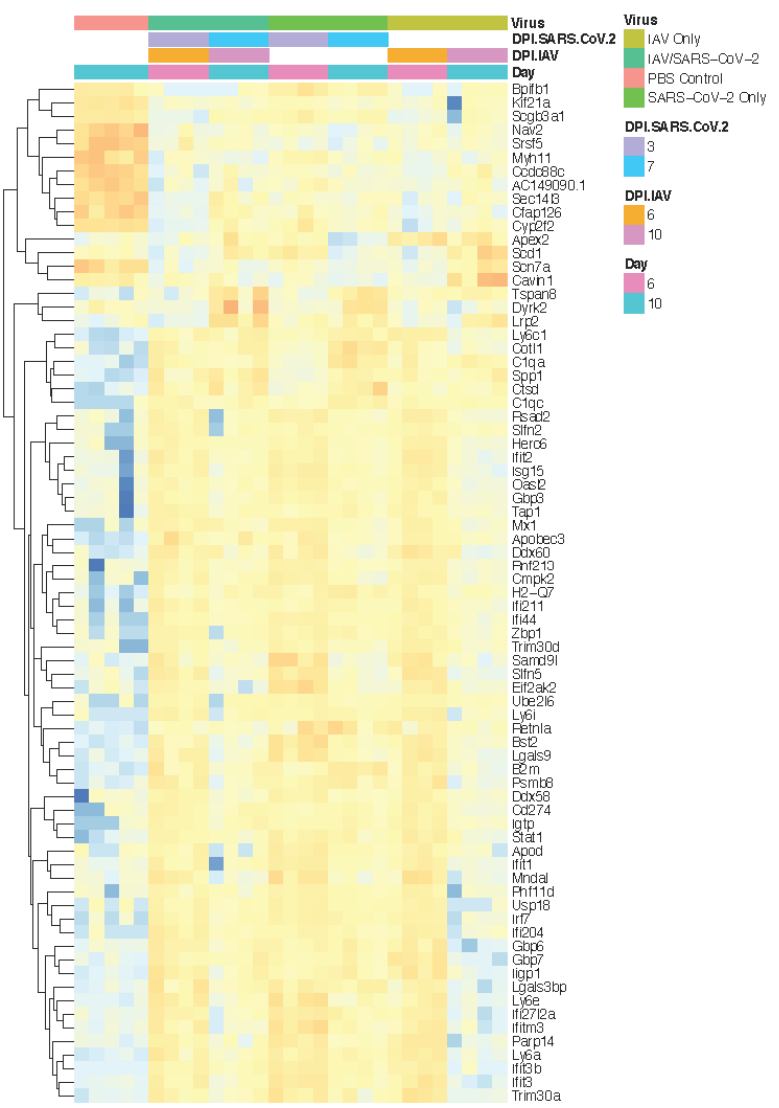

C Day 6 Day 10
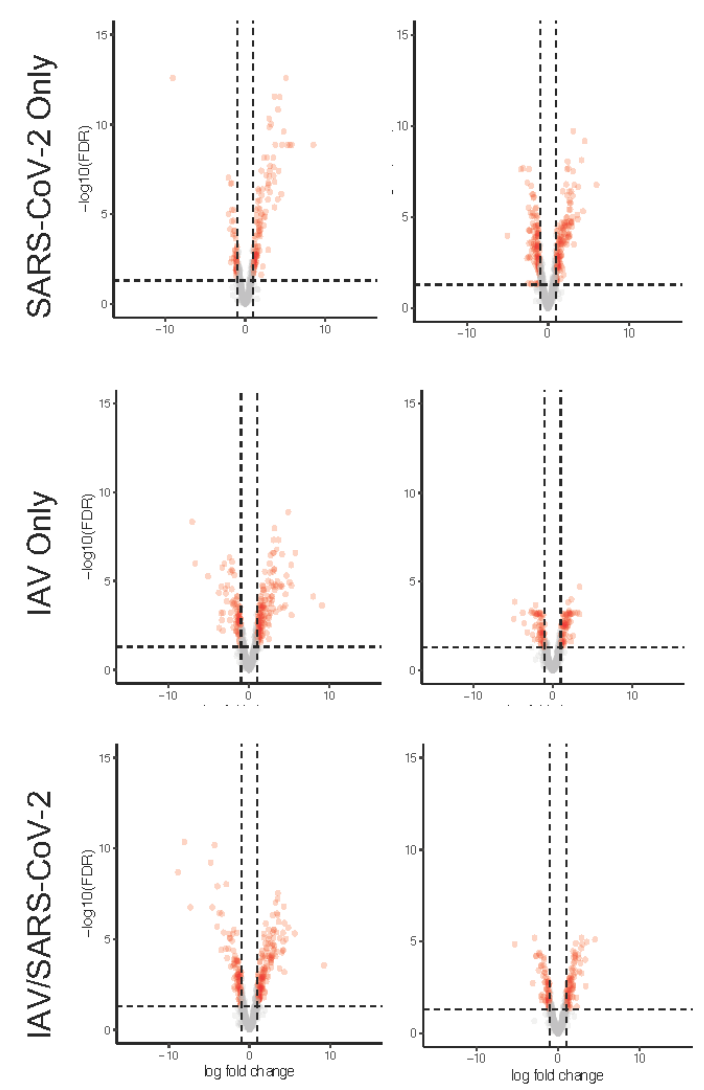
Figure 10: RNA sequencing analysis from hACE2 mice lung homogenates from mice infected with either IAV only, SARS-CoV-2 only or IAV and SARS-CoV-2 ( $n=4-5)$. A. Principle component analysis performed for 29 samples with log2 transformed counts per million (cpm). B. The top 75 differentially expressed gene transcripts across 4 groups are shown. C. Volcano plots comparing differentially expressed genes from each infection group vs mock infected. The horizontal dashed line is representative of a q-value $<0.05$, and the vertical dashed line is representative of a log 2 fold-change of 2 . Significant differentially expressed gene transcripts are marked as red.

Gene ontology analysis of gene transcripts that were significantly different in abundance at all time points revealed enrichment of gene clusters involved in the innate immune response, immune system regulation and cellular response to cytokine stimulus, interferon beta and interferon gamma (supplementary Fig. S5). The differentially expressed gene transcripts between coinfection day 10 versus IAV day 10 were associated with interferon responses according to biological process terms (supplementary Fig. S6).

\section{Interferon and cytokine responses are upregulated in response to infection, and maintained in coinfection}

Following gene ontology analysis, gene transcripts were grouped by biological process terms and presented as heatmaps to allow for direct comparison of their abundance across the experimental groups. SARS-CoV-2 infection resulted in the increased abundance of gene transcripts involved in the interferon and cytokine signalling pathways. When mice were infected with both SARS-CoV-2 and IAV, certain gene transcripts within these pathways remained increased in abundance at later time points, in comparison to individual IAV infection at day 10 (Fig. 11). These included Ifit1, Ifit3, Ifit3b, Isg15, Irf7 and Cxcl10. This suggested a sustained innate/interferon response in these animals. 


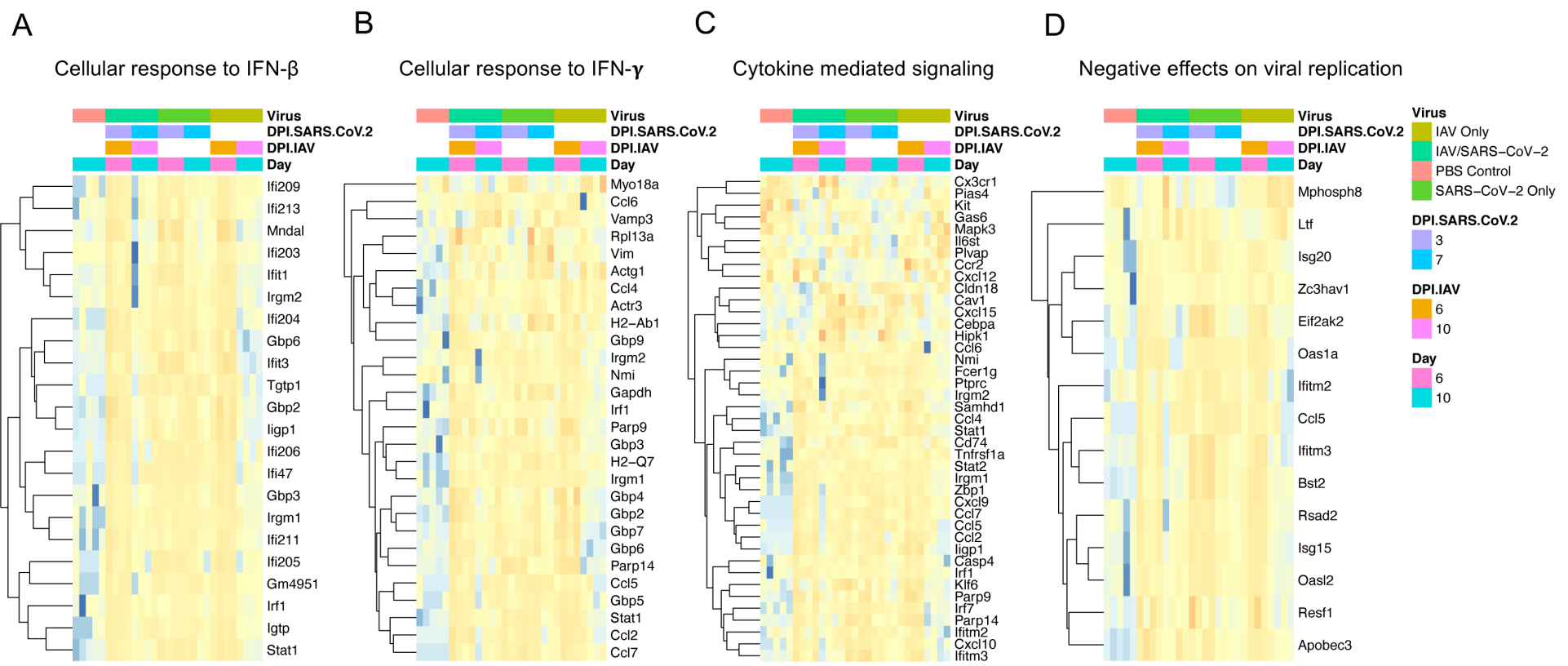

Figure 11: Heatmaps showing expression of genes enriched in gene ontology terms. A. Cellular response to IFN- $\beta$ (GO:0035458). B. Cellular response to IFN- $\gamma$ (GO:0071346). C. Cytokine mediated signalling (GO:0019221). D. Negative effects on viral replication (GO:0045071).

\section{Discussion}

In this study, sequential infection with IAV followed by SARS-CoV-2 led to more severe pulmonary disease than infections with IAV or SARS-CoV-2 alone. Following IAV infection, mice coinfected with SARS-CoV-2 displayed significantly higher weight loss, elevated respiratory distress and more rapid mortality compared to mice infected with IAV alone. Transcriptomics analysis revealed that the expression of several genes specific to airway epithelial cells such as Scgb3a1, Cfap126, and Cyp2f2 was more downregulated in coinfected mice compared to singly infected mice at days 6 and 10, which is indicative of increased lung epithelial cell damage. Interestingly, coinfected mice exhibited significantly lower levels of SARS-CoV-2 viral RNA and sgRNA at day 6 (3 dpi with SARS-CoV-2) compared to SARS-CoV-2 singly infected mice, indicating that, whilst coinfection results in enhanced respiratory signs, existing IAV infection interferes with infection of SARS-CoV-2 at this time point. These findings were recapitulated by analysis and comparison of the pathological changes in the lungs. Overt pulmonary damage was due to IAV and was represented by multifocal epithelial 
cell necrosis in bronchioles and adjacent alveoli. SARS-CoV-2 infection was also multifocal but only affected alveoli distant from those affected by IAV, consistent with infection via aerosol from the upper respiratory tract and reflecting that both viruses compete for their target cells in alveoli; accordingly, destruction of alveoli by IAV could explain the lower SARS-CoV-2 loads in double infected mice. By day 10, coinfected mice and SARS-CoV-2 singly infected mice displayed similar levels of viral RNA and sgRNA, suggesting that whilst initially inhibited by the presence of IAV, SARS-CoV-2 was able to overcome this inhibition and achieve unconstrained viral replication. This was reflected in the lung transcriptome profile that showed a sustained innate response in coinfected animals over the time-period of both infections. Histological examination showed that at 10 days post infection, the damage induced by IAV was resolving. There were distinct regenerative changes, represented by respiratory epithelial cell and type II pneumocyte hyperplasia, accompanied by a moderate macrophage dominated inflammatory response. SARS-CoV-2 infection still showed the same distribution pattern, with viral antigen expression in epithelial cells of unaltered appearing alveoli, but not in hyperplastic type II cells confirming that, like other coronaviruses, also SARS-CoV-2 infects only fully differentiated epithelial cells. However, there was evidence of SARS-CoV-2 induced damage, represented by syncytia formation of type II pneumocytes and more pronounced alveolar damage (desquamative pneumonia and occasional hyaline membrane formation). With SARSCoV-2 single infection the inflammatory response appears to be less macrophage dominated, as T cells are present in similar amoounts.

Viral Interference is a well-documented phenomenon which has previously been reported between influenza $B$ viruses (IBV) in a ferret model, in which infection with one IBV subtype was able to prevent infection with another subtype when infections were separated by 3 days ${ }^{27}$. Similar to the observations described herein between IAV and SARS-CoV-2, coinfection of antigenically unrelated viruses such as IAV and IBV did not confer resistance when challenged within 3 days, but merely delayed the shedding of the challenged virus. This delay in shedding likely accounts for the differing times at which coinfected animals and SARS-CoV-2 singly infected animals lose weight; SARS-CoV-2 singly infected animals exhibit weight loss at 4dpi however, coinfected animals begin to recover from IAV infection before succumbing to delayed SARS-CoV-2 infection. Unlike this study, wherein IAV and SARS-CoV-2 coinfected 
animals exhibited significantly increased weight loss, coinfection with IAV and IBV has been reported to lead to delayed viral shedding but did not influence disease severity.

Mathematical modelling and in vitro and in vivo studies have shown that prior infection with rhinovirus interferes with IAV infection ${ }^{5}$. This interference is mediated by the induction of interferon stimulated genes (ISGs) following rhinovirus infection which work to suppress IAV. Similarly, infection with IAV results in the activation of the IFN response and the upregulation of ISGs which induce an antiviral state which works to limit infection ${ }^{28}$ and reviewed ${ }^{29}$. We propose that it is this response which is active in the $\mathrm{K}-18 \mathrm{hACE} 2$ mice 3 days post IAV infection and is responsible for the inhibition of the incoming SARS-CoV-2 infection, thus resulting in lower viral load as measured by RT-qPCR at day 6. IAV viral load was found to be similar between coinfected and IAV singly infected mice, demonstrating that SARS-CoV-2 infection does not interfere with prior IAV infection. Similarly, by day 10 (7 dpi SARS-CoV-2) both coinfected and IAV singly infected mice were negative for IAV by immunohistology and qPCR, indicating that SARS-CoV-2 infection does not prolong IAV infection or interfere with the ability of the immune system to clear IAV infection. At this stage, the IAV singly infected mice exhibited histological features consistent with regeneration including hyperplasia of the bronchiolar epithelium and type II pneumocytes. This finding was supported by the transcriptomic profiles of the IAV singly infected mice at day 10 which showed overlap with the healthy controls. These transcriptomic profiles were characterised by the decreased expression of several ISGs including Isg15, Ligp1, Gbp6, Ifi206, Ifitm3 and Ifit3, compared to day 6. Conversely, while the coinfected mice also displayed evidence of epithelial regeneration, they also presented several hallmarks of acute lung injury including perivascular infiltration, vasculitis and oedema. This elevated lung injury is consistent with the viral loads of SARS-CoV-2 present in these animals at day 10 and the lung transcriptomic profile which revealed that the coinfected mice maintained heightened levels of ISG transcription in addition to several other genes associated with cytokine and IFN-Y signalling compared to the IAV singly infected mice.

As has recently been reported in studies using K18-hACE2 transgenic mice ${ }^{23,30}$, some of the SARS-CoV-2 singly infected and coinfected mice had developed a nonsuppurative meningoencephalitis by day 10 , predominantly affecting the midbrain and 
brainstem. Previous studies using K18-hACE2 mice focusing on SARS-CoV have shown that the virus spreads throughout the brain around 3 days post intranasal inoculation $21,23,31$. Our study does not provide evidence of viral spread to the brain at this early stage; however, distribution of virus antigen and inflammatory changes are consistent with ascending infection from the nasal cavity, via the olfactory bulb ${ }^{31}$ while the other showed little involvement ${ }^{21}$. Unlike SARS-CoV-2, SARS-CoV has been shown to enter the brain earlier at $3 \mathrm{dpi}$ but does not elicit notable inflammation in this secondary site of infection ${ }^{31}$. Interestingly, the coinfected mice displayed more substantial virus spread in the brain and a more pronounced perivascular infiltration with evidence of structural blood-brain-barrier (BBB) breakdown. The mechanism through which co-infection with IAV may enhance SARS-CoV-2 neurological infection is unclear. While brain infection has been well documented in cases of influenza ${ }^{32-34}$, this is predominantly limited to neurotropic and highly pathogenic strains and occurs via breakdown of the BBB following high levels of viremia ${ }^{33,35}$. BBB integrity is also reduced by proinflammatory cytokines such as IL-6, IL-1 $\beta$ and IFN- $\gamma$ which disrupt the tight-junctions maintained by brain microvascular endothelial cells (reviewed in ${ }^{36}$ ). While the IAV X31 strain used herein did not result in brain infection, it is possible that the increased cytokine response present in coinfected animals further compromised the BBB integrity and allowed better access of SARS-CoV-2 to the brain.

No animal model can predict with absolute certainty the consequences of coinfection in humans. However, the data presented here may have critical implications for development of successful pre-emptive interventions for SARS-CoV-2. Fortunately, public health interventions aimed at delaying the transmission of SARS-CoV-2 should also provide a consequent reduction in transmission of influenza if they are effectively implemented. Moreover, some but not all experimental therapeutics being studied for SARS-CoV-2 have also been demonstrated to exhibit activity against influenza. As for other viruses for which successful antiviral interventions have been developed, the SARS-CoV-2 polymerase has emerged as a strong initial target for small molecule inhibitors. Importantly, drugs such as remdesivir and favipiravir that are in various stages of development and clinical evaluation for SARS-CoV-2 have a direct or metabolite-driven in vitro activity against influenza ${ }^{37,38}$, with favipiravir also approved for influenza in Japan. Other examples of dual activity against these viruses are evident with other small molecule antivirals such as nitazoxanide ${ }^{39-41}$ and niclosamide 
42,43 , which may present opportunities and/or a basis for prioritisation of candidates for clinical evaluation if necessary exposures can be achieved 44,45 . Such antiviral interventions have potential application in treatment of early infection as well as the prophylactic setting. Chemoprevention is a particularly attractive approach as we move into winter months, and selection of the right candidates for evaluation may provide a benefit for both viruses individually and in coinfection. It should be noted that many of the advanced technologies (e.g. broadly neutralising monoclonal antibodies) that are being rapidly accelerated through development have explicit specificities that provide high potency, but this is likely to preclude activity against viruses other than those against which they are directed. The work presented here shows that our approach will be an effective pre-clinical platform with which to test therapeutic approaches to dealing with co-infection which is pertinent with the approaching flu season in the Northern hemisphere concomitant with a second wave of SARS-CoV-2 infections. 


\section{Acknowledgements:}

This work was funded by the US Food and Drug Administration Grant, Characterization of severe coronavirus infection in humans and model systems for medical countermeasure development and evaluation, to $\mathrm{JAH}$, and by the Biotechnology and Biological Sciences Research Council (BBSRC) grants BB/R00904X/1 and BB/R018863/1 to JPS. R. P.-R. was supported by a PhD studentship from the MRC Discovery Medicine North (DiMeN) Doctoral Training Partnership (MR/N013840/1). LT is supported by the Wellcome Trust (grant number 205228/Z/16/Z) and the National Institute for Health Research Health Protection Research Unit (NIHR HPRU) in Emerging and Zoonotic Infections (NIHR200907) at University of Liverpool in partnership with Public Health England (PHE), in collaboration with Liverpool School of Tropical Medicine and the University of Oxford. LT is based at University of Liverpool. The views expressed are those of the author(s) and not necessarily those of the NHS, the NIHR, the Department of Health or Public Health England. 


\section{Methods}

\section{Cell culture and virus}

Influenza virus A/HKx31 (X31, H3N2) was propagated in the allantoic cavity of 9-dayold embryonated chicken eggs at $35^{\circ} \mathrm{C}$ for $72 \mathrm{~h}$. Titres were determined by an influenza plaque assay using MDCK cells.

Vero E6 cells (C1008; African green monkey kidney cells) were obtained from Public Health England and maintained in Dulbecco's minimal essential medium (DMEM) containing $10 \%$ foetal bovine serum (FBS) and $0.05 \mathrm{mg} / \mathrm{mL}$ gentamycin at $37^{\circ} \mathrm{C}$ with $5 \% \mathrm{CO}_{2}$.

UK strain of SARS-CoV-2 (hCoV-2/human/Liverpool/REMRQ0001/2020), which was cultured from a nasopharyngeal swab from a patient, was passaged a further 4 times in Vero E6 cells ${ }^{24}$. The fourth passage of virus was cultured $\mathrm{MOI}$ of 0.001 in Vero E6 cells with DMEM containing 4\% FBS and $0.05 \mathrm{mg} / \mathrm{mL}$ gentamycin at $37^{\circ} \mathrm{C}$ with $5 \% \mathrm{CO}_{2}$ and was harvested $48 \mathrm{~h}$ post inoculation. Virus stocks were stored at $-80^{\circ} \mathrm{C}$. The intracellular viral genome sequence and the titre of virus in the supernatant were determined. Direct RNA sequencing was performed as describe previously ${ }^{25}$ and an inhouse script was used to check for deletions in the mapped reads. The Illumina reads were mapped to the England/2/2020 genome using HISAT and the consensus genome was called using an in-house script based on the dominant nucleotide at each location on the genome. The sequence has been submitted to Genbank, accession number MW041156.

\section{Ethics and clinical information}

The patient from which the virus sample was obtained gave informed consent and was recruited under the International Severe Acute Respiratory and emerging Infection Consortium (ISARIC) Clinical Characterisation Protocol CCP (https://isaric.net/ccp), reviewed and approved by the national research ethics service, Oxford (13/SC/0149). Samples from clinical specimens were processed at containment level 3 at the University of Liverpool.

Biosafety. All work was performed in accordance with risk assessments and standard operating procedures approved by the University of Liverpool Biohazards SubCommittee and by the UK Health and Safety Executive. Work with SARS-CoV-2 was 
performed at containment level 3 by personnel equipped with respirator airstream units with filtered air supply.

\section{Mice}

Animal work was approved by the local University of Liverpool Animal Welfare and Ethical Review Body and performed under UK Home Office Project Licence PP4715265. Mice carrying the human ACE2 gene under the control of the keratin 18 promoter (K18-hACE2; formally B6.Cg-Tg(K18-ACE2)2PrImn/J) were purchased from Jackson Laboratories. Mice were maintained under SPF barrier conditions in individually ventilated cages.

\section{Virus infection}

Animals were randomly assigned into multiple cohorts. For IAV infection, mice were anaesthetized lightly with KETASET i.m. and inoculated intra-nasally with $10^{2}$ PFU IAV X31 in $50 \mu$ sterile PBS. For SARS-CoV-2 infection, mice were anaesthetized lightly with isoflurane and inoculated intra-nasally with $50 \mu$ l containing $10^{4}$ PFU SARSCoV-2 in PBS. They were sacrificed at variable time-points after infection by an overdose of pentabarbitone. Tissues were removed immediately for downstream processing.

\section{Histology, immunohistology}

The left lung, kidneys, liver and brain were fixed in 10\% neutral buffered formalin for 24-48 $\mathrm{h}$ and routinely paraffin wax embedded. Consecutive sections (3-5 $\mu \mathrm{m})$ were either stained with haematoxylin and eosin (HE) or used for immunohistology $(\mathrm{IH})$. IH was performed to detect viral antigens and ACE2 expression and to identify macrophages, T cells and B cells using the horseradish peroxidase (HRP) method. The following primary antibodies were applied: rabbit anti-human ACE2 (Novus Biologicals; clone SN0754; NBP2-67692), goat anti-IAV (Meridian Life Sciences Inc., B65141G), rabbit anti-lba-1 (antigen: AIF1; Wako Chemicals; macrophage marker), mAB rabbit anti-mouse CD3 (clone SP7: Spring Bioscience, Ventana Medical Systems, Tucson, USA; T cell marker) and rat anti-mouse CD45R (clone B220, BD Biosciences; B cell marker), following previously published protocols ${ }^{46-48}$. and rabbit anti-SARS-CoV nucleocapsid protein (Rockland, 200-402-A50). Briefly, after deparaffination, sections underwent antigen retrieval in citrate buffer ( $\mathrm{pH} \mathrm{6.0;} \mathrm{Agilent)}$ 
for ACE2 and SARS-CoV NP detection, and Tris/EDTA buffer (pH 9) for IAV for 20 min at $98^{\circ} \mathrm{C}$, followed by incubation with the primary antibodies (diluted in dilution buffer, Agilent; anti-IAV 1:200, anti-ACE2, 1:200 and anti-SARS-CoV 1:3000) and overnight at $4^{\circ} \mathrm{C}$ for ACE2 and SARS-CoV and for $1 \mathrm{~h}$ at room temperature (RT) for IAV. This was followed by blocking of endogenous peroxidase (peroxidase block, Agilent) for $10 \mathrm{~min}$ at RT and incubation with the secondary antibodies, EnVision+/HRP, Rabbit (Agilent) for ACE2 and SARS-CoV, and rabbit anti-goat Ig/HRP (Agilent) for IAV), for 30 min at RT, and EnVision FLEX DAB+ Chromogen in Substrate buffer (Agilent) for $10 \mathrm{~min}$ at RT, all in an autostainer (Dako). Sections were subsequently counterstained with haematoxylin.

In addition, four mock infected transgenic control mice were subjected to a phenotyping, examining upper and lower airways, lungs, kidneys, liver, small intestine, spleen and brain for any histological changes and for ACE2 expression.

Lungs and brain from an age-matched wild type BALB-C mouse stained for ACE2 served to assess any differences in the ACE expression pattern in the transgenic mice, and two wild type C57BL6/J mice infected intranasally with $10^{2}$ PFU IAV X31 in $50 \mu$ I sterile PBS and sacrificed at 6 days post infection served to assess any effect of hACE2 expression in the course of IAV infection. A formalin-fixed, paraffin embedded cell pellet infected with IAV for $24 \mathrm{~h}$ served as positive control for the IAV staining, and the spleen of a control mouse as positive control for the leukocyte markers.

\section{RNA extraction and DNase treatment}

The upper lobes of the right lung were dissected and homogenised in $1 \mathrm{ml}$ of TRIzol reagent (Thermofisher) using a Bead Ruptor 24 (Omni International) at 2 meters per second for $30 \mathrm{sec}$. The homogenates were clarified by centrifugation at 12,000xg for 5 min before full RNA extraction was carried out according to manufacturer's instructions. RNA was quantified and quality assessed using a Nanodrop (Thermofisher) before a total of $1 \mathrm{ug}$ was DNase treated using the TURBO DNA-free ${ }^{\mathrm{TM}}$ Kit (Thermofisher) as per manufacturer's instructions.

\section{qRT-PCR for viral load}

Viral loads were quantified using the GoTaq® Probe 1-Step RT-qPCR System (Promega). For quantification of SARS-COV-2 the nCOV_N1 primer/probe mix from the SARS-CoV-2 (2019-nCoV) CDC qPCR Probe Assay (IDT) were utilised while the 
standard curve was generated via 10-fold serial dilution of the 2019-nCoV_N_Positive Control (IDT) from $10^{6}$ to 0.1 copies/reaction. The E sgRNA primers and probe have been previously described (https://www.nature.com/articles/s41586-020-2196-x) and were utilised at $400 \mathrm{nM}$ and $200 \mathrm{nM}$ respectively. Murine $18 \mathrm{~S}$ primers and probe sequences ere utilsied at 400nM and 200nM respectively. The IAV primers and probe sequences are published as part of the CDC IAV detection kit (20403211). The IAV reverse genetics plasmid encoding the NS segment was diluted 10 -fold from $10^{6}$ to 0.1 copies/reaction to serve as a standard curve. The thermal cycling conditions for all qRT-PCR reactions were as follows: 1 cycle of $45^{\circ} \mathrm{C}$ for $15 \mathrm{~min}$ and 1 cycle of $95^{\circ} \mathrm{C}$ followed by 40 cycles of $95^{\circ} \mathrm{C}$ for $15 \mathrm{sec}$ and $60^{\circ} \mathrm{C}$ for 1 minute. The $18 \mathrm{~s}$ standard was generated by the amplification of a fragment of the murine 18S cDNA using the primers F: ACCTGGTTGATCCTGCCAGGTAGC and R: GCATGCCAGAGTCTCGTTCG. Similarly, the E sgRNA standard was generated by PCR using the qPCR primers. cDNA was generated using the SuperScript IV reverse transcriptase kit (Thermofisher) and PCR carried out using Q5® High-Fidelity 2X Master Mix (New England Biolabs) as per manufacturer's instructions. Both PCR products were purified using the QIAquick PCR Purification Kit (Qiagen) and serially diluted 10-fold from $10^{10}$ to $10^{4}$ copies/reaction to form the standard curve.

\section{cDNA sequencing with Oxford Nanopore}

cDNA libraries were made starting with 50ng of total RNA which was accurately quantified using a Qubit 3.0 fluorometer (Thermofisher) and the Qubit RNA HS Assay Kit (Thermofisher). The cDNA was generated using the PCR-cDNA Barcoding (SQKPCB109) sequencing kit by Oxford Nanopore Technologies. Multiplexed libraries were loaded onto a R9.4.1 flowcell and ran for up to 72 hours on a GridION. The raw sequence reads have been submitted to NCBI SRA under bioproject: PRJNA666048.

\section{RNA sequencing bioinformatic analysis}

Multiplexed sequencing reads were basecalled and demultiplexed by guppy. Minimap2 was used to index and map reads to the reference genome (Mus_musculus.GRCm38.cdna.all.fa) to generate alignment files using the -ax mapont -N 100 -p 1.0 parameters ${ }^{49}$. Alignment files were sorted and indexed with samtools before counting reads using Salmon with the corresponding annotation file (Mus_musculus.GRCm38.gtf) from Ensembl using -noErrorModel -I U parameters 
26,50. The edgeR package was used to normalise sequencing libraries and identify differentially expressed genes, defined as at least a 2-fold difference from the mock infected group $(n=5)$ and a false discovery rate (FDR) less than $0.05^{51}$. Principle component Analysis (PCA), volcano plots, heatmaps and Venn diagrams were produced in $\mathrm{R}$ studio using the following packages: edgeR, ggplot2 and pheatmap. Differential gene expression data was used for gene ontology enrichment analysis of biological process terms in each group using enrichGO in the ClusterProfiler programme in $R{ }^{52}$. A q-value cut-off of 0.05 was used with a Benjamini-HochbergFDR correction. GOSemSim was used to simplify and remove redundant GO terms ${ }^{53}$ and the top 20 biological processes are presented for each condition.

Statistical analysis. Data were analysed using the Prism package (version 5.04 Graphpad Software). $P$ values were set at $95 \%$ confidence interval. A repeatedmeasures two-way ANOVA (Bonferroni post-test) was used for time-courses of weight loss; two-way ANOVA (Bonferroni post-test) was used for other time-courses; log-rank (Mantel-Cox) test was used for survival curves. All differences not specifically stated to be significant were not significant $(p>0.05)$. For all figures, ${ }^{*} p<0.05,{ }^{* *} p<0.01$, ${ }^{* * *} p<0.001$. 


\section{References}

$1 \mathrm{Ng}$, L. F. P. \& Hiscox, J. A. Coronaviruses in animals and humans. BMJ 368, m634, doi:10.1136/bmj.m634 (2020).

2 Channappanavar, R. \& Perlman, S. Pathogenic human coronavirus infections: causes and consequences of cytokine storm and immunopathology. Semin Immunopathol 39, 529-539, doi:10.1007/s00281-017-0629-x (2017).

3 Alosaimi, B. et al. MERS-CoV infection is associated with downregulation of genes encoding Th1 and Th2 cytokines/chemokines and elevated inflammatory innate immune response in the lower respiratory tract. Cytokine 126, 154895, doi:10.1016/j.cyto.2019.154895 (2020).

4 Dorward, D. A. et al. Tissue-specific tolerance in fatal Covid-19. medRxiv, 2020.2007.2002.20145003, doi:10.1101/2020.07.02.20145003 (2020).

$5 \quad$ Nickbakhsh, S. et al. Virus-virus interactions impact the population dynamics of influenza and the common cold. Proc Natl Acad Sci $U S A$, doi:10.1073/pnas.1911083116 (2019).

6 Morens, D. M., Taubenberger, J. K. \& Fauci, A. S. Predominant role of bacterial pneumonia as a cause of death in pandemic influenza: implications for pandemic influenza preparedness. J Infect Dis 198, 962-970, doi:10.1086/591708 (2008).

7 Carroll, M. W. et al. Deep Sequencing of RNA from Blood and Oral Swab Samples Reveals the Presence of Nucleic Acid from a Number of Pathogens in Patients with Acute Ebola Virus Disease and Is Consistent with Bacterial Translocation across the Gut. mSphere 2, doi:10.1128/mSphereDirect.0032517 (2017).

8 Gaunt, E. R., Hardie, A., Claas, E. C., Simmonds, P. \& Templeton, K. E. Epidemiology and clinical presentations of the four human coronaviruses 229E, HKU1, NL63, and OC43 detected over 3 years using a novel multiplex real-time PCR method. J Clin Microbiol 48, 2940-2947, doi:10.1128/JCM.00636-10 (2010).

9 Lee, N. et al. Co-circulation of human metapneumovirus and SARS-associated coronavirus during a major nosocomial SARS outbreak in Hong Kong. J Clin Virol 40, 333-337, doi:10.1016/j.jcv.2007.08.015 (2007).

10 Alfaraj, S. H., Al-Tawfiq, J. A., Alzahrani, N. A., Altwaijri, T. A. \& Memish, Z. A. The impact of co-infection of influenza A virus on the severity of Middle East Respiratory Syndrome Coronavirus. J Infect 74, 521-523, doi:10.1016/j.jinf.2017.02.001 (2017).

11 Du, Y. et al. Clinical Features of 85 Fatal Cases of COVID-19 from Wuhan. A Retrospective Observational Study. Am J Respir Crit Care Med 201, 13721379, doi:10.1164/rccm.202003-05430C (2020).

12 Ma, S., Lai, X., Chen, Z., Tu, S. \& Qin, K. Clinical characteristics of critically ill patients co-infected with SARS-CoV-2 and the influenza virus in Wuhan, China. Int J Infect Dis 96, 683-687, doi:10.1016/j.ijid.2020.05.068 (2020).

13 Azekawa, S., Namkoong, H., Mitamura, K., Kawaoka, Y. \& Saito, F. Coinfection with SARS-CoV-2 and influenza A virus. IDCases 20, e00775, doi:10.1016/j.idcr.2020.e00775 (2020).

14 Yue, $\mathrm{H}$. et al. The epidemiology and clinical characteristics of co-infection of SARS-CoV-2 and influenza viruses in patients during COVID-19 outbreak. $J$ Med Virol, doi:10.1002/jmv.26163 (2020). 
15 Kondo, Y., Miyazaki, S., Yamashita, R. \& Ikeda, T. Coinfection with SARS-CoV2 and influenza A virus. BMJ Case Rep 13, doi:10.1136/bcr-2020-236812 (2020).

16 Hashemi, S. A., Safamanesh, S., Ghasemzadeh-Moghaddam, H., Ghafouri, M. \& Azimian, A. High prevalence of SARS-CoV-2 and influenza A virus (H1N1) coinfection in dead patients in Northeastern Iran. $J$ Med Virol, doi:10.1002/jmv.26364 (2020).

17 Hashemi, S. A. et al. Co-infection with COVID-19 and influenza A virus in two died patients with acute respiratory syndrome, Bojnurd, Iran. J Med Virol, doi:10.1002/jmv.26014 (2020).

18 Stowe, J. et al. Interactions between SARS-CoV-2 and Influenza and the impact of coinfection on disease severity: A test negative design. medRxiv, 2020.2009.2018.20189647, doi:10.1101/2020.09.18.20189647 (2020).

19 Ami, Y. et al. Co-infection of respiratory bacterium with severe acute respiratory syndrome coronavirus induces an exacerbated pneumonia in mice. Microbiol Immunol 52, 118-127, doi:10.1111/j.1348-0421.2008.00011.x (2008).

20 Ryan, K. A. et al. Dose-dependent response to infection with SARS-CoV-2 in the ferret model: evidence of protection to re-challenge. bioRxiv, 2020.2005.2029.123810, doi:10.1101/2020.05.29.123810 (2020).

21 McCray, P. B., Jr. et al. Lethal infection of K18-hACE2 mice infected with severe acute respiratory syndrome coronavirus. $J$ Virol 81, 813-821, doi:10.1128/JVI.02012-06 (2007).

22 Oladunni, F. S. et al. Lethality of SARS-CoV-2 infection in K18 human angiotensin converting enzyme 2 transgenic mice. bioRxiv, 2020.2007.2018.210179, doi:10.1101/2020.07.18.210179 (2020).

23 Winkler, E. S. et al. SARS-CoV-2 infection of human ACE2-transgenic mice causes severe lung inflammation and impaired function. Nat Immunol, doi:10.1038/s41590-020-0778-2 (2020).

24 Patterson, E. I. et al. Methods of Inactivation of SARS-CoV-2 for Downstream Biological Assays. J Infect Dis 222, 1462-1467, doi:10.1093/infdis/jiaa507 (2020).

25 Davidson, A. D. et al. Characterisation of the transcriptome and proteome of SARS-CoV-2 reveals a cell passage induced in-frame deletion of the furin-like cleavage site from the spike glycoprotein. Genome Med 12, 68, doi:10.1186/s13073-020-00763-0 (2020).

26 Patro, R., Duggal, G., Love, M. I., Irizarry, R. A. \& Kingsford, C. Salmon provides fast and bias-aware quantification of transcript expression. Nat Methods 14, 417-419, doi:10.1038/nmeth.4197 (2017).

27 Laurie, K. L. et al. Evidence for Viral Interference and Cross-reactive Protective Immunity Between Influenza B Virus Lineages. J Infect Dis 217, 548-559, doi:10.1093/infdis/jix509 (2018).

28 Forero, A. et al. Evaluation of the innate immune responses to influenza and live-attenuated influenza vaccine infection in primary differentiated human nasal epithelial cells. Vaccine 35, 6112-6121, doi:10.1016/j.vaccine.2017.09.058 (2017).

29 Killip, M. J., Fodor, E. \& Randall, R. E. Influenza virus activation of the interferon system. Virus Res 209, 11-22, doi:10.1016/j.virusres.2015.02.003 (2015).

30 Lutz, C., Maher, L., Lee, C. \& Kang, W. COVID-19 preclinical models: human angiotensin-converting enzyme 2 transgenic mice. Hum Genomics 14, 20, doi:10.1186/s40246-020-00272-6 (2020). 
31 Netland, J., Meyerholz, D. K., Moore, S., Cassell, M. \& Perlman, S. Severe acute respiratory syndrome coronavirus infection causes neuronal death in the absence of encephalitis in mice transgenic for human ACE2. $J$ Virol 82, 72647275, doi:10.1128/JVI.00737-08 (2008).

32 Hosseini, S. et al. Long-Term Neuroinflammation Induced by Influenza A Virus Infection and the Impact on Hippocampal Neuron Morphology and Function. $J$ Neurosci 38, 3060-3080, doi:10.1523/JNEUROSCI.1740-17.2018 (2018).

33 Chaves, A. J. et al. Neuroinvasion of the highly pathogenic influenza virus H7N1 is caused by disruption of the blood brain barrier in an avian model. PLoS One 9, e115138, doi:10.1371/journal.pone.0115138 (2014).

34 Ekstrand, J. J. Neurologic complications of influenza. Semin Pediatr Neurol 19, 96-100, doi:10.1016/j.spen.2012.02.004 (2012).

35 Wang, S. et al. Influenza virus-cytokine-protease cycle in the pathogenesis of vascular hyperpermeability in severe influenza. J Infect Dis 202, 991-1001, doi:10.1086/656044 (2010).

36 Miner, J. J. \& Diamond, M. S. Mechanisms of restriction of viral neuroinvasion at the blood-brain barrier. Curr Opin Immunol 38, 18-23, doi:10.1016/j.coi.2015.10.008 (2016).

37 Baranovich, T. et al. T-705 (favipiravir) induces lethal mutagenesis in influenza A H1N1 viruses in vitro. J Virol 87, 3741-3751, doi:10.1128/JVI.02346-12 (2013).

38 Cho, A. et al. Synthesis and antiviral activity of a series of 1'-substituted 4-aza7,9-dideazaadenosine C-nucleosides. Bioorg Med Chem Lett 22, 2705-2707, doi:10.1016/j.bmcl.2012.02.105 (2012).

39 Wang, M. et al. Remdesivir and chloroquine effectively inhibit the recently emerged novel coronavirus (2019-nCoV) in vitro. Cell Res 30, 269-271, doi:10.1038/s41422-020-0282-0 (2020).

40 Belardo, G., Cenciarelli, O., La Frazia, S., Rossignol, J. F. \& Santoro, M. G. Synergistic effect of nitazoxanide with neuraminidase inhibitors against influenza A viruses in vitro. Antimicrob Agents Chemother 59, 1061-1069, doi:10.1128/AAC.03947-14 (2015).

41 Haffizulla, J. et al. Effect of nitazoxanide in adults and adolescents with acute uncomplicated influenza: a double-blind, randomised, placebo-controlled, phase $2 \mathrm{~b} / 3$ trial. Lancet Infect Dis 14, 609-618, doi:10.1016/S14733099(14)70717-0 (2014).

42 Jurgeit, A. et al. Niclosamide is a proton carrier and targets acidic endosomes with broad antiviral effects. PLoS Pathog 8, e1002976, doi:10.1371/journal.ppat.1002976 (2012).

43 Jeon, S. et al. Identification of Antiviral Drug Candidates against SARS-CoV-2 from FDA-Approved Drugs. Antimicrob Agents Chemother 64, doi:10.1128/AAC.00819-20 (2020).

44 Arshad, U. et al. Prioritization of Anti-SARS-Cov-2 Drug Repurposing Opportunities Based on Plasma and Target Site Concentrations Derived from their Established Human Pharmacokinetics. Clin Pharmacol Ther 108, 775790, doi:10.1002/cpt.1909 (2020).

45 Rajoli, R. K. et al. Dose prediction for repurposing nitazoxanide in SARS-CoV2 treatment or chemoprophylaxis. medRxiv, doi:10.1101/2020.05.01.20087130 (2020).

46 Schmid, A. S., Hemmerle, T., Pretto, F., Kipar, A. \& Neri, D. Antibody-based targeted delivery of interleukin-4 synergizes with dexamethasone for the 
reduction of inflammation in arthritis. Rheumatology (Oxford). 57, 748-755, doi:10.1093/rheumatology/kex447 (2018).

47 Hughes, D. J. et al. Chemokine binding protein M3 of murine gammaherpesvirus 68 modulates the host response to infection in a natural host. PLoS Pathog 7, e1001321, doi:10.1371/journal.ppat.1001321 (2011).

48 Calabrese, F. et al. Herpes virus infection is associated with vascular remodeling and pulmonary hypertension in idiopathic pulmonary fibrosis. PLoS One 8, e55715, doi:10.1371/journal.pone.0055715 (2013).

$49 \mathrm{Li}, \mathrm{H}$. Minimap2: pairwise alignment for nucleotide sequences. Bioinformatics 34, 3094-3100, doi:10.1093/bioinformatics/bty191 (2018).

$50 \mathrm{Li}, \mathrm{H}$. et al. The Sequence Alignment/Map format and SAMtools. Bioinformatics 25, 2078-2079, doi:10.1093/bioinformatics/btp352 (2009).

51 Robinson, M. D., McCarthy, D. J. \& Smyth, G. K. edgeR: a Bioconductor package for differential expression analysis of digital gene expression data. Bioinformatics 26, 139-140, doi:10.1093/bioinformatics/btp616 (2010).

52 Yu, G., Wang, L. G., Han, Y. \& He, Q. Y. clusterProfiler: an R package for comparing biological themes among gene clusters. OMICS 16, 284-287, doi:10.1089/omi.2011.0118 (2012).

$53 \mathrm{Yu}, \mathrm{G}$. Gene Ontology Semantic Similarity Analysis Using GOSemSim. Methods Mol Biol 2117, 207-215, doi:10.1007/978-1-0716-0301-7_11 (2020). 


\section{Supplementary figures}

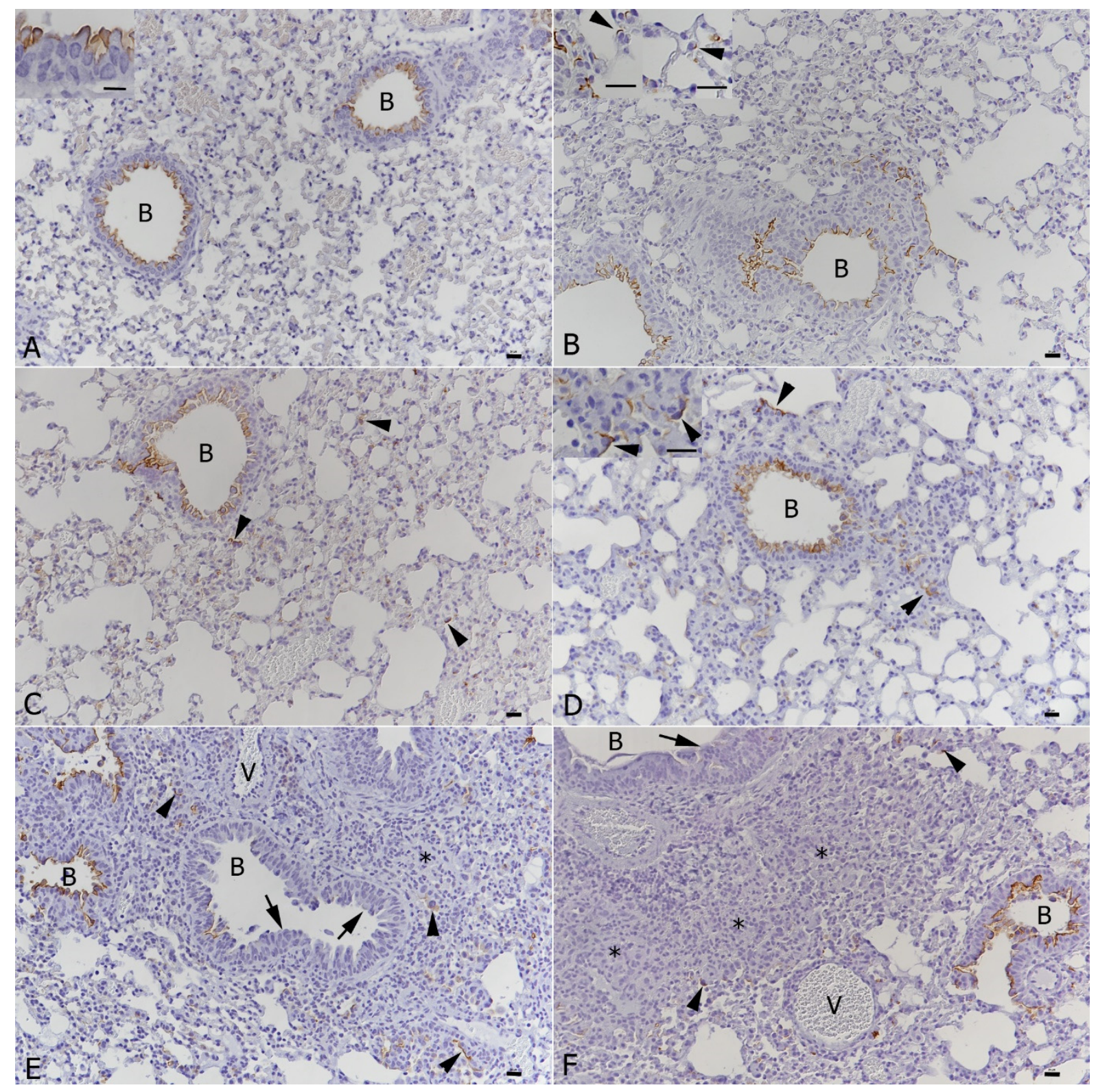

Supplementary Figure S1. ACE2 expression in the lungs. A. Wild type mouse, uninfected. The epithelium lining the bronchioles (B) shows ACE2 expression at the luminal surface (inset). B. Mock infected K18-hACE2 mouse. Besides the epithelium lining the bronchioles (B), there are scattered type II pneumocytes that express ACE2 (insets: arrowheads). C. K18hACE2 mouse, 3 days post SARS-CoV-2 infection. The expression pattern and extent is comparable to that in the mock infected K18-hACE2 mouse. Arrowheads point at ACE2 positive type II pneumocytes. D. K18-hACE2 mouse, 7 days post SARS-CoV-2 infection. The expression pattern is identical to that in the mock infected K18-hACE2 mouse, but activated type II pneumocytes that express ACE2 (arrowheads) are more numerous. E. K18-hACE2 mouse, 10 days post IAV infection. In unaltered bronchioles, the ACE2 expression pattern is identical to that in bronchioles of the mock infected K18-hACE2 mouse. However, in bronchioles with epithelial hyperplasia, the hyperplastic epithelium appears ACE2 negative (arrows). Also in areas of type II respiratory epithelial metaplasia/type II pneumocyte hyperplasia ( $\left.{ }^{*}\right)$, the cells are ACE2 negative. There is also a mild increase in ACE2 positive type II pneumocytes (arrowheads). F. Double infected K18-hACE2 mouse, 10 days post IAV infection and 7 days post SARS-CoV-2 infection. Similar to the single IAV infected animal at 
$10 \mathrm{dpi}$, there is ACE2 expression in the unaltered bronchiolar epithelium, while it is absent in the hyperplastic bronchiolar epithelium and in areas of respiratory epithelial metaplasia/type II pneumocyte hyperplasia $\left({ }^{*}\right)$. Positive type II pneumocytes (arrowheads) are also observed.

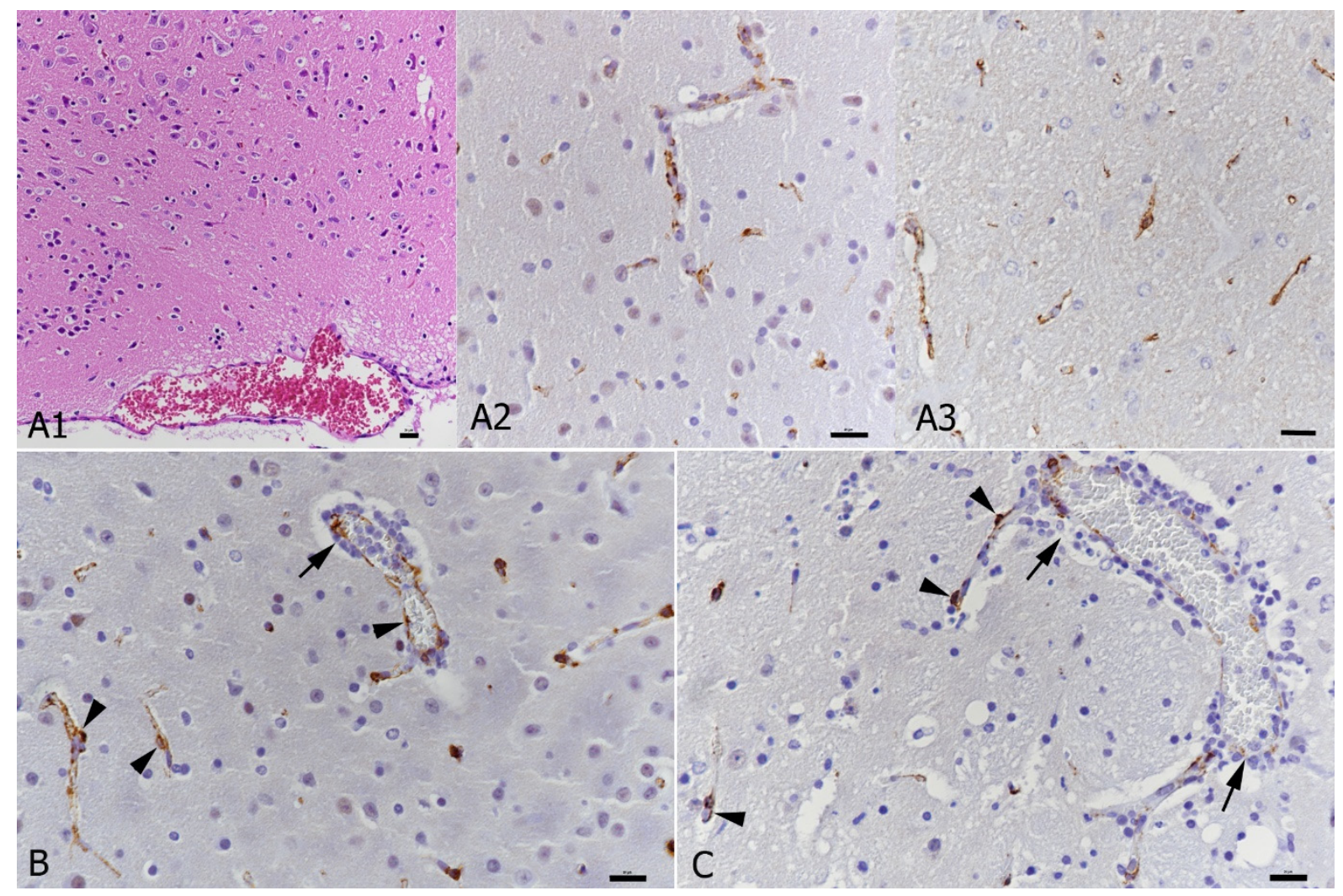

Supplementary Figure S2. ACE2 expression in the brain. A. Mock infected control animals. A1. K18-hACE2 transgenic mouse, unaltered hypothalamus. A2, A3. ACE2 expression in capillary endothelial cells in K18-hACE2 transgenic mouse (A2) and wildtype mouse (A3). B. SARS-CoV-2 infected hACE transgenic mouse. Endothelial ACE2 staining shows an intact endothelial layer (arrowheads) also in areas of perivascular infiltration (arrow). C. IAV and SARS-CoV-2 double infected hACE transgenic mouse. Endothelial ACE2 expression (arrowheads) is lost in areas of more intense infiltration (arrows). HE stain and immunohistology, hematoxylin counterstain. Bars represent $20 \mu \mathrm{m}$. 
Day 6
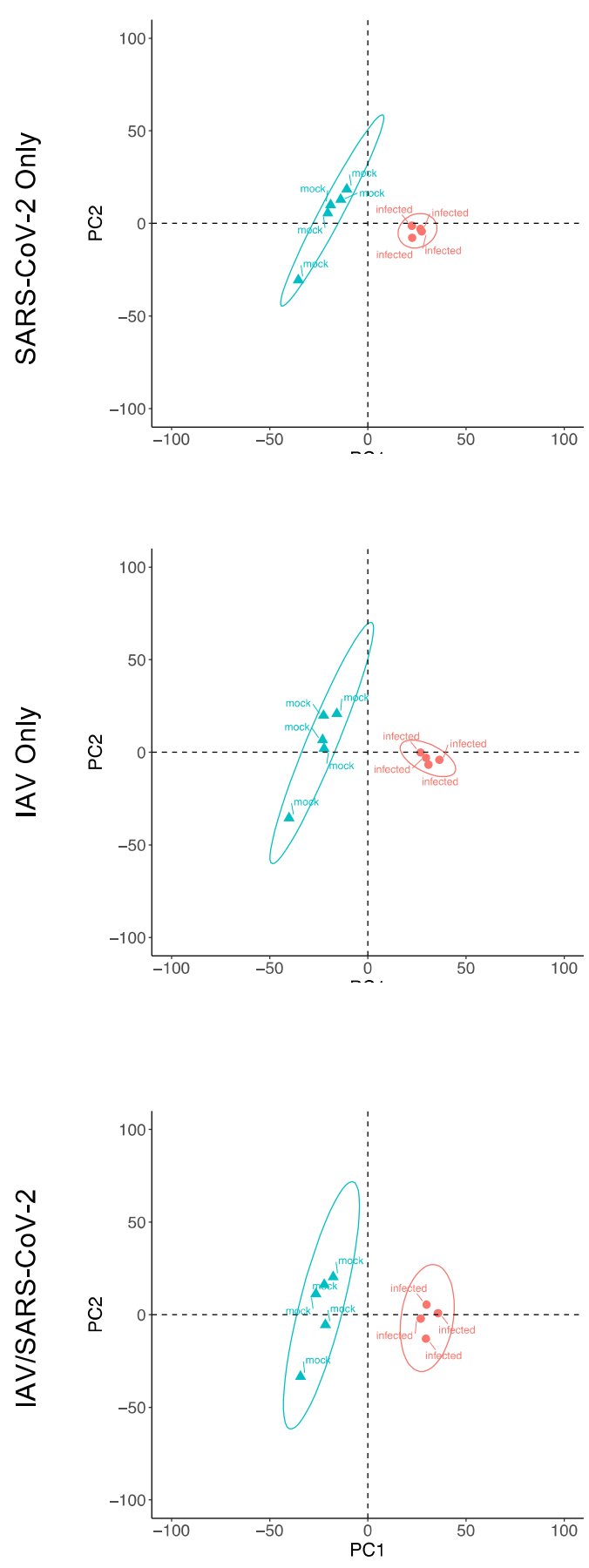

Day 10
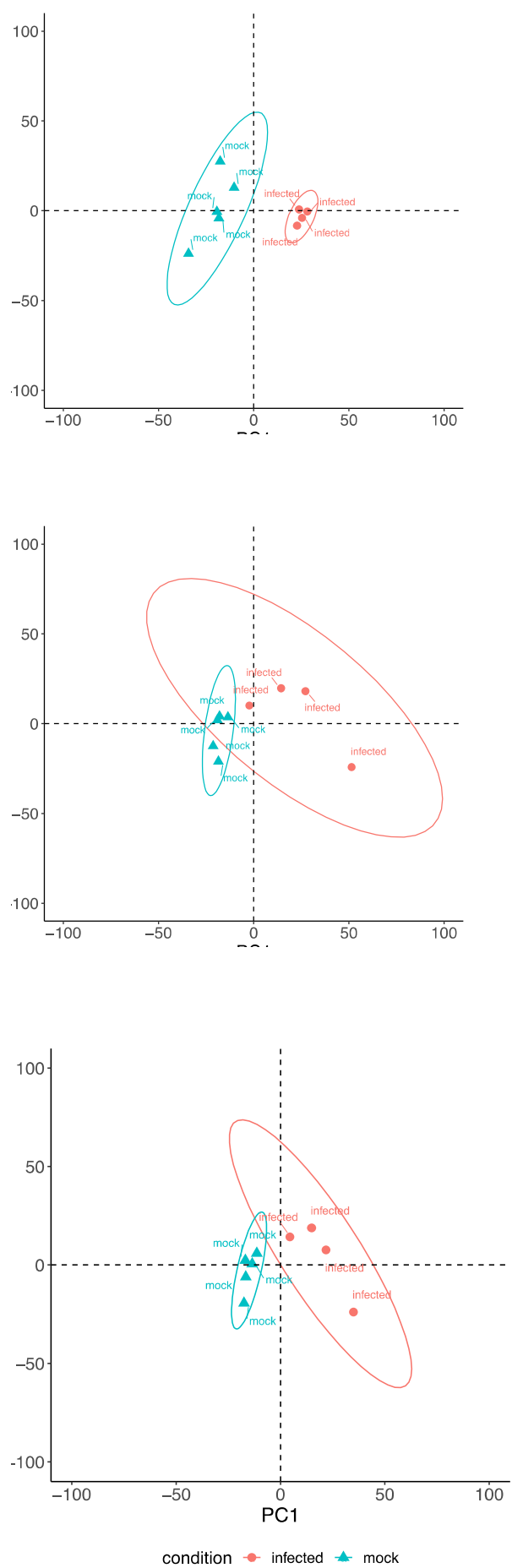

Supplementary Figure S3: RNA sequencing analysis from hACE2 mice lung homogenates from mice infected with either IAV only, SARS-CoV-2 only or IAV and SARS-CoV-2 ( $n=4-$ 5). Each plot shows a principle component analysis performed for 9 samples with log2 transformed counts per million (cpm). 5 mock infected mice compared with 4 infected mice at day 6 and 10. 
bioRxiv preprint doi: https://doi.org/10.1101/2020.10.13.334532; this version posted February 15, 2021. The copyright holder for this

preprint (which was not certified by peer review) is the author/funder, who has granted bioRxiv a license to display the preprint in perpetuity. It is made available under aCC-BY 4.0 International license.

SARS-CoV-2 Day 3

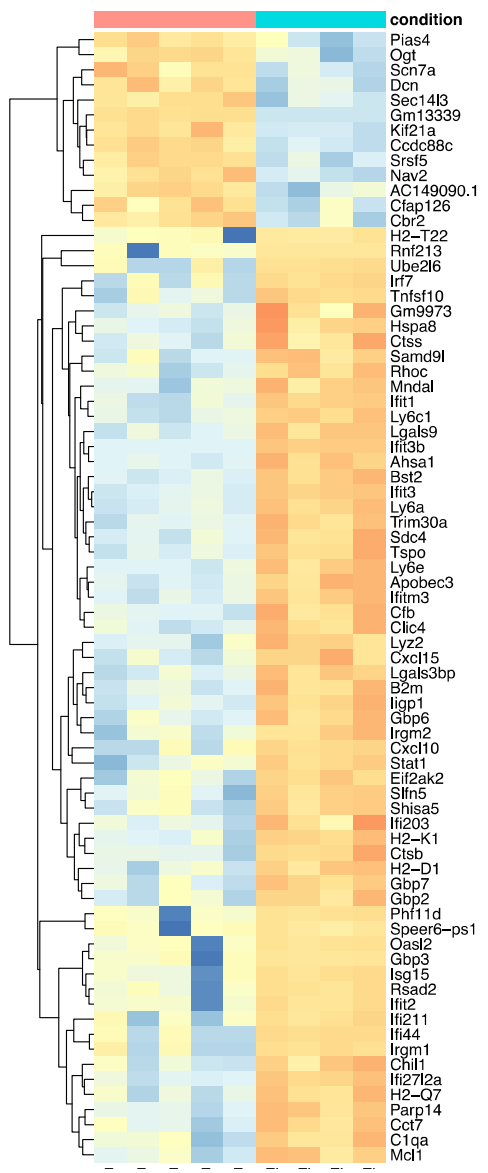

IAV Day 6

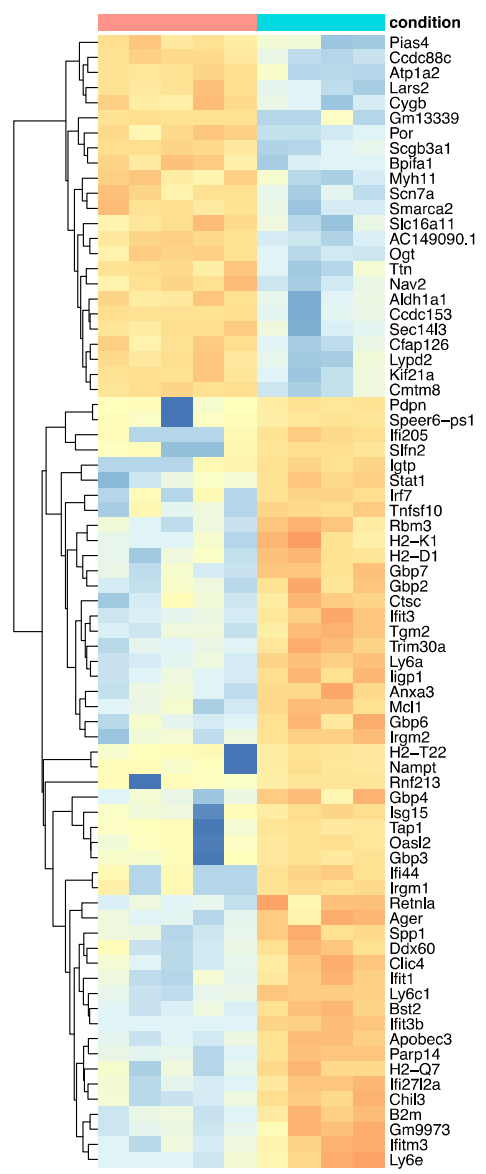

\section{SARS-CoV-2 Day 7}

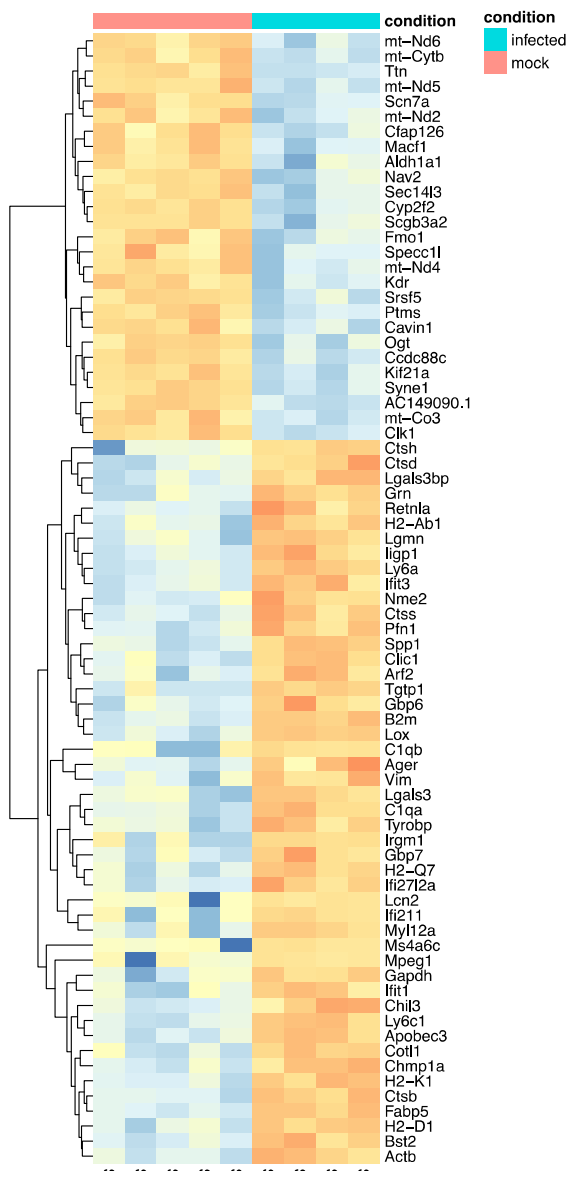

IAV Day 10

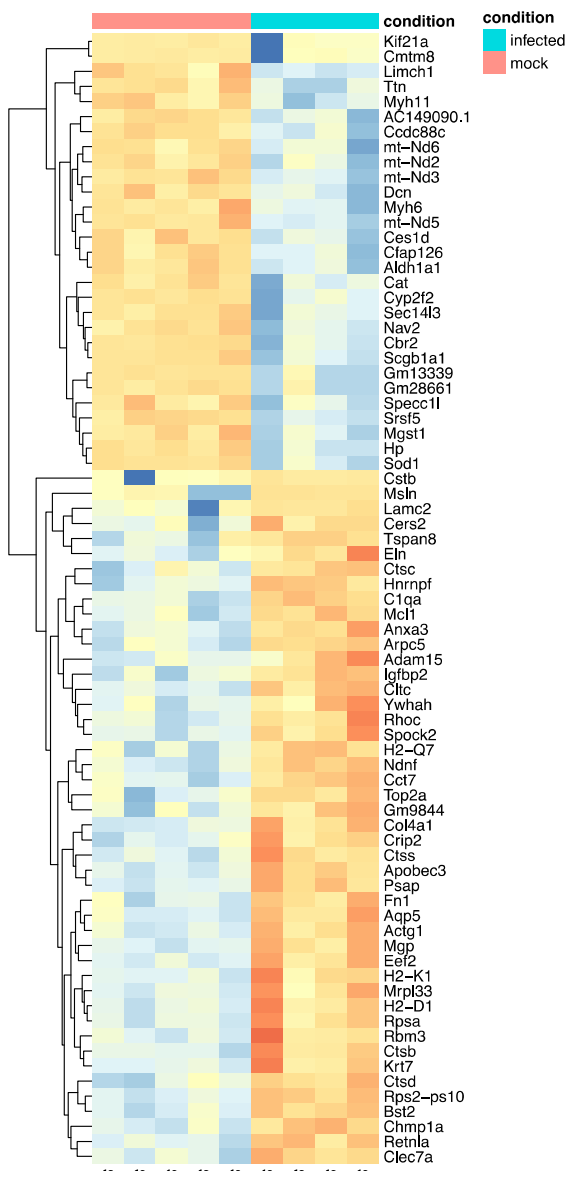



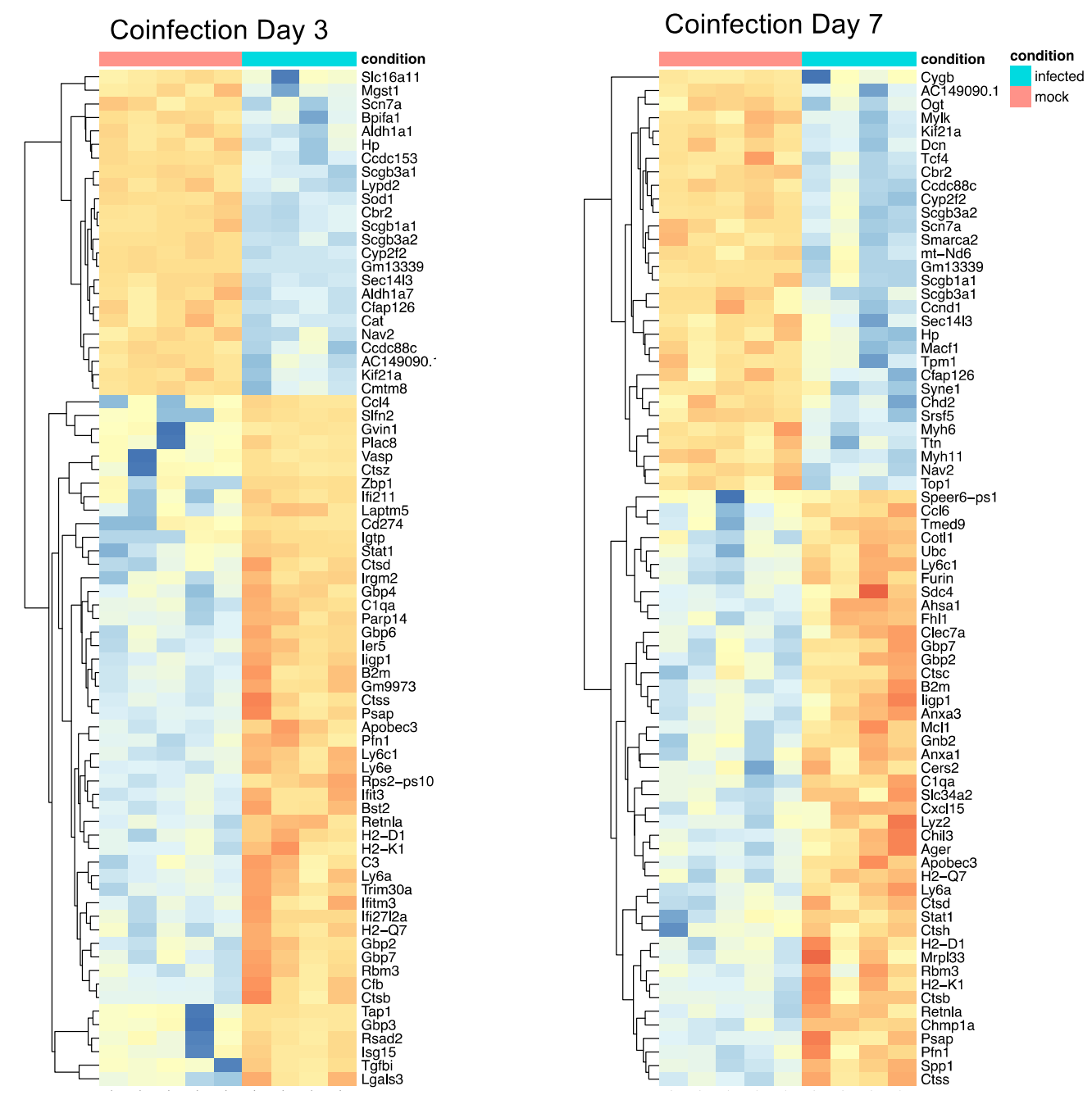

Supplementary figure S4: RNA sequencing analysis from hACE2 mice lung homogenates from mice infected with either IAV only, SARS-CoV-2 only or IAV and SARS-CoV-2 ( $n=4-5)$. Each heatmap presents the top 75 differentially expressed gene transcripts for each infection type (SARS-CoV-2 only, IAV only or IAV and SARS-CoV-2) compared to mock infected mice $(n=5)$ at day 6 and day $10(n=4)$ is shown individually. 

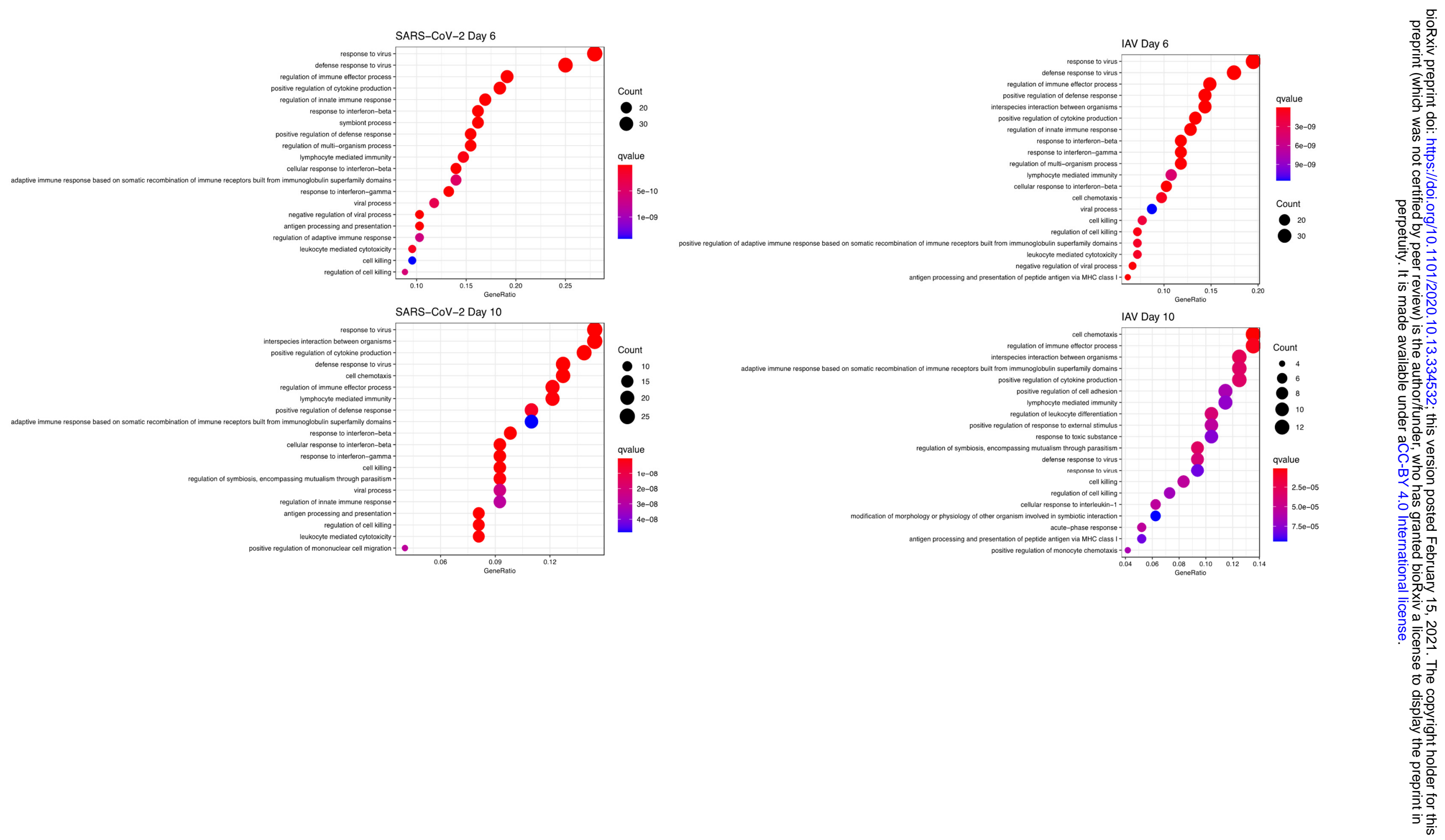


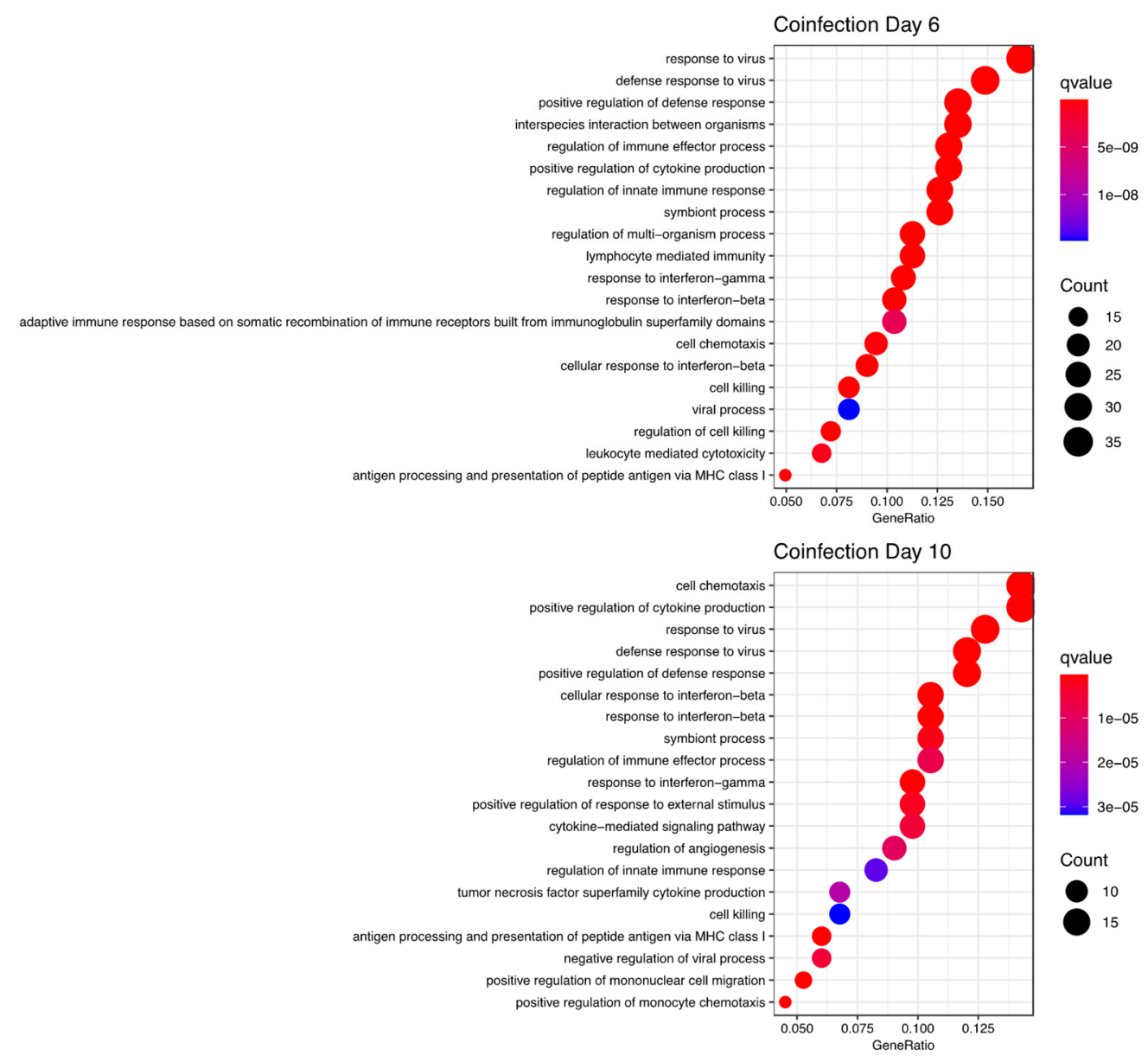

Supplementary figure S5: Gene ontology enrichment analysis of biological process terms enriched in differentially expressed genes in comparison to mock infected mice. The top 20 terms are presented in a dotplot. Counts of genes within the cluster are represented by the size of the dot, and the false-discovery rate (qvalue) is highlighted by colour.

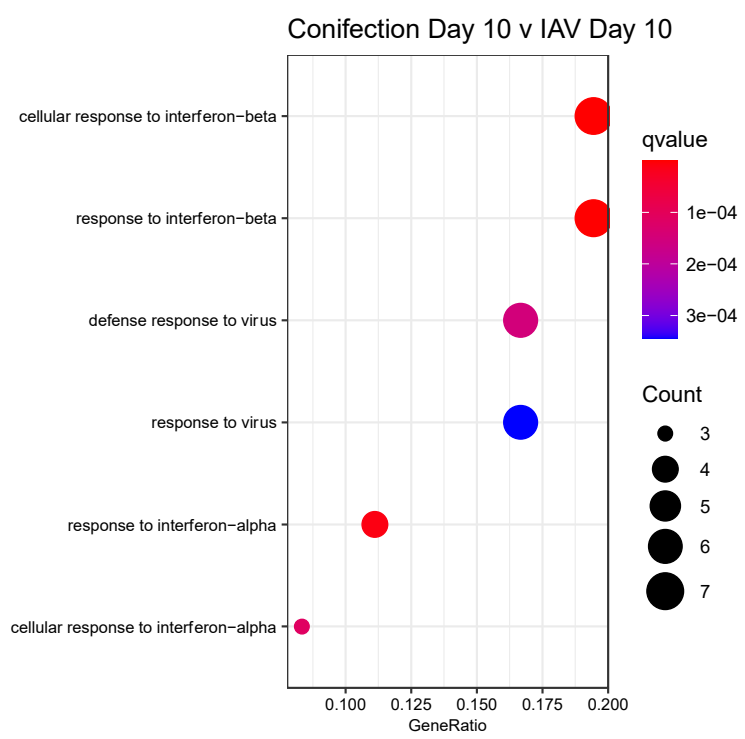

Supplementary figure S6: Gene ontology enrichment analysis of biological process terms enriched in Coinfection day 10 in comparison to IAV day 10. 